\title{
Direct Reconstruction of Pharmacokinetic-Rate Images of Optical Fluorophores From NIR Measurements
}

\author{
Burak Alacam and Birsen Yazıc1*, Senior Member, IEEE
}

\begin{abstract}
In this paper, we present a new method to form pharmacokinetic-rate images of optical fluorophores directly from near infra-red (NIR) boundary measurements. We first derive a mapping from spatially resolved pharmacokinetic rates to NIR boundary measurements by combining compartmental modeling with a diffusion based NIR photon propagation model. We express this mapping as a state-space equation. Next, we introduce a spatio-temporal prior model for the pharmacokinetic-rate images and combine it with the state-space equation. We address the image formation problem using the extended Kalman filtering framework. We analyze the computational complexity of the resulting algorithms and evaluate their performance in numerical simulations. An important feature of our approach is that the reconstruction of fluorescence concentrations and compartmental modeling are combined into a single step 1) to take advantage of the inherent temporal correlations in dynamic NIR measurements, and 2) to incorporate spatio-temporal a priori information on pharmacokinetic-rate images. Simulation results show that the resulting algorithms are more robust and lead to higher signal-to-noise ratio as compared to existing approaches where the reconstruction of concentrations and compartmental modeling are treated separately. Additionally, we reconstructed pharmacokinetic-rate images using in vivo data obtained from three patients with breast tumors. The reconstruction results show that the pharmacokinetic rates of indocyanine green are higher inside the tumor region as compared to the surrounding tissue.
\end{abstract}

Index Terms-Compartmental analysis, direct reconstruction, extended Kalman filter, indocyanine green, pharmacokinetics.

\section{INTRODUCTION}

\section{A. Motivation and Overview of Our Approach}

$\mathbf{P}$ HARMACOKINETICS can be defined as the mathematics of the time course of absorption, distribution, and excretion of fluorophores, contrast agents, and drugs in the body [1]. The biological alterations such as tumor development

Manuscript received October 28, 2008; revised February 01, 2009. First published February 27, 2009; current version published August 26, 2009. This work was supported by U.S. Army Medical Research Acquisition Activity under Grant W81XWH-04-1-0559 and in part by the Center for Subsurface Sensing and Imaging Systems, under the Engineering Research Centers Program of the National Science Foundation under Award Number EEC-9986821. Asterisk indicates corresponding author.

B. Alacam is with Department of Electrical and Electronics Engineering, Middle East Technical University, Northern Cyprus Campus, Northern Cyprus (e-mail: alacam@metu.edu.tr).

*B. Yazıcı is with the Department of Electrical, Computer, and Systems Engineering and the Department of Biomedical Engineering, Rensselaer Polytechnic Institute, Troy, NY 12180 USA (e-mail: yazici@ecse.rpi.edu).

Color versions of one or more of the figures in this paper are available online at http://ieeexplore.ieee.org

Digital Object Identifier 10.1109/TMI.2009.2015294 which influence the transfer process of fluorophores or drugs also influence the rate of change of absorption and excretion in the body. Hence, pharmacokinetic analysis of fluorophores is a potential means for tumor detection, diagnosis, and staging; treatment monitoring; and drug delivery and feasibility studies.

Compartmental modeling is a well-known approach in pharmacokinetic analysis [2]-[4]. In this method, a region of interest is decomposed into a number of compartments, each representing a volume of tissue in which the fluorophore distribution may be similar. The concentration change in a specific compartment is modeled as a result of the exchange of fluorophores between connected compartments. These changes are modeled by a collection of coupled ordinary differential equations (ODE); each equation describing the fluorophore exchanges between the interacting compartments. The coefficients of the ODE's represent the rates of exchange between different compartments and are called the pharmacokinetic rates.

In this paper, we are interested in imaging the pharmacokinetic rates of optical fluorophores using near infrared (NIR) diffuse optical tomographic methods. As in magnetic resonance imaging [5]-[7] and positron emission tomography [8]-[13], pharmacokinetic rate analysis of optical fluorophores can provide valuable physiological information for tumor detection, diagnosis, and staging; treatment monitoring; and drug delivery and feasibility studies.

In the NIR range, the most widely used optical fluorophore is indocyanine green (ICG) [14]-[16]. ICG is a U.S. Food and Drug Administration approved NIR absorbing and fluorescing dye. It is an intravascular contrast agent that may extravasate through vessels of high permeability, such as cancerous vessels. Therefore, ICG pharmacokinetic-rate imaging of the tumor region mainly probes permeability and vascularization. Several research groups, including our group, have recently reported compartmental modeling of ICG in animal and human subjects and studied the parameters related to capillary permeability as malignancy indicators [17]-[20]. In all these studies, the pharmacokinetic rates are assumed to be constant over a tissue volume that may be as large as the entire imaging domain.

Recently, we reported ICG pharmacokinetic-rate images of three patients with breast tumors and demonstrated that the spatially resolved pharmacokinetic rates may provide superior information than a single set of rates obtained for the entire breast for cancer diagnosis [21]. We obtained ICG pharmacokinetic images in two steps. First, we reconstructed the ICG concentration images based on the differential diffuse optical tomographic forward model. Next, we fit a compartmental model to the time 
sequence of voxels for each physical location. Therefore, we refer to this algorithm as the voxel-by-voxel algorithm.

In this paper, we develop a new method to reconstruct spatially resolved pharmacokinetic rates of fluorophores directly from dynamic NIR boundary measurements. We first derive a mathematical model that maps the boundary measurements to the total fluorophore concentrations. We call this map concentration-to-measurement (CTM) map. For this, we combine the compartmental model with an NIR photon propagation model which maps the total fluorophore concentrations to boundary measurements. To model photon propagation, we use the diffusion approximation to radiative transfer equation where the propagation of excitation and emission light are modeled by two coupled diffusion equations [30]. We derive two CTM maps: linear time-invariant CTM map and nonlinear time-varying CTM map for dynamic boundary measurements. In the linear time-invariant CTM map, the relationship between the total fluorophore concentration and the boundary measurements is linear and does not vary during the entire tomographic data collection process. In the nonlinear time-varying CTM map, on the other hand, the relationship between the total fluorophore concentration and the boundary measurements is nonlinear at every instant of the tomographic data collection process and it varies during the data collection process. We form a state-space model based on the compartmental model equations and the NIR photon propagation model. We, then, derive a map that relates the boundary measurements to spatially resolved pharmacokinetic rates. We call this mapping pharmacokinetic-rates-to-measurement (PTM) map. We introduce a spatio-temporal prior model for the pharmacokinetic rate and volume fraction parameters and incorporate this model to the state-space representation. We addressed the resulting image reconstruction problem by the extended Kalman filtering (EKF) framework.

We analyze the computational complexity of the resulting algorithms (linear and nonlinear) and compare them with that of the voxel-by-voxel algorithm [21]. We evaluate the performance of our algorithms in numerical simulations using a tissue like numerical phantom. Our numerical study shows that there is a good agreement between the true and the estimated images in terms of localization of the heterogeneities and with respect to normalized mean-squared error criterion. Furthermore, the numerical studies show that the new method is more robust than the voxel-by-voxel algorithm with higher signal-tonoise ratio. Additionally, we present reconstruction of pharmacokinetic-rate images from in vivo data acquired from three patients with breast tumors. The reconstructed images show that the pharmacokinetic rates are higher inside the tumor region as compared to the surrounding tissue.

\section{B. Related Work and the Advantages of Our Approach}

EKF framework was previously used to address the reconstruction of optical parameters [22]-[24]. In [22], Eppstein et al. utilized EKF to reconstruct images of absorption coefficient, fluorescence lifetime, and quantum efficiency using simulated noisy measurements. This study uses a dynamic model to impose spatial smoothing on the unknown optical parameters, but does not consider dynamic imaging of time-varying optical parameters. In [23] and [24], Kolehmainen et al., and Prince et al. used EKF to reconstruct time-varying absorption images of human motor cortex. These studies demonstrate that EKF provides an effective framework in dynamic tomography problems in diffuse optical imaging. The underlying dynamic model in these studies assume that the unknown optical image, i.e., the state variables, remains constant up to an additive noise term and the measurement model is given by the diffusion equation. In our work, on the other hand, the dynamic model is based on the coupled ODE's of the compartmental model, while the measurement model is based on the CTM map derived from the two coupled diffusion equation representing the fluorescence light propagation.

An important feature of the method introduced here is the direct reconstruction of the pharmacokinetic-rate images of optical fluorophores as opposed to voxel-by-voxel reconstruction algorithm that we reported in [21]. While the voxel-by-voxel algorithm can be modified to take into account spatial correlations in total concentration images, it does not take into account the temporal correlations present in dynamically changing fluorophore concentrations due to the decoupled nature of the two-step algorithm described above. The new method, on the other hand, makes effective use of the temporal correlations present in the dynamic measurements by coupling the two steps and incorporating a spatio-temporal a priori model. This has the advantage of improved robustness and signal-to-noise ratio as compared to the voxel-by-voxel reconstruction method.

In [25], Milstein et al. presented a direct reconstruction method for the pharmacokinetic-rate images of optical fluorophores. This work uses an exponential curve model for compartmental modeling and a linear time-invariant fluorescence light propagation model derived based on authors' prior work in [26] and [27]. The reconstruction of pharmacokinetic-rate images is addressed based on the maximum $a$ posteriori (MAP) estimation together with a parametric iterative coordinate descent optimization technique similar to the approach reported in [13].

Our work has the following advantages as compared to the other direct reconstruction methods: 1) We use coupled ODEs with a model mismatch term for compartmental modeling. This has the advantage of providing better fit than the exponential curve models for modeling the pharmacokinetics of optical fluorophores. 2) Our compartmental modeling and reconstruction technique is not specific to a particular optical fluorophore, but applicable to the pharmacokinetic-rate imaging of any optical fluorophore. 3) Unlike the methods in [18], [19], and [25] our method estimates the pharmacokinetic-rate parameters, volume fractions, and concentrations in different compartments explicitly. 4) Our method addresses the pharmacokinetic-rate imaging based on both linear time-invariant and nonlinear time-varying CTM maps. Nonlinear time-varying CTM map provides a more accurate relationship between the total fluorophore concentration and boundary measurements. 5) MAP estimation-based approach requires an explicit solution for the coupled ODEs used for the compartmental modeling. These solutions are often obtained under some assumptions which often limit the applicability of the resulting compartmental model. EKF-based approach, on the other hand, can accommodate stochastic coupled ODEs, eliminating any limitations on the applicability 
of the compartmental model. 6) While MAP inverse problem formulation can accommodate arbitrary non-Gaussian prior models and provides theoretically better noise suppression, the noise suppression property of Gaussian-based EKF can be improved by backward smoothing [28] or by adapting a non-Gaussian approach to EKF [29]. 7) As compared to the nonlinear least squares curve fitting techniques, the EKF-based approach can accommodate spatio-temporal prior models. 8) The EKF-based reconstruction techniques can be used for real-time pharmacokinetic-rate imaging.

\section{Organization of the Paper}

The rest of the paper is organized as follows. Section II describes the spatially resolved compartmental modeling and the two-compartment model for ICG pharmacokinetics. In Section III, we present the NIR photon propagation model based on coupled diffusion equations. In Section IV, we present the pharmacokinetic-rate-to-measurement map for optical fluorophores. In Section V, we present the spatio-temporal prior model, the method of direct reconstruction of pharmacokinetic-rate images based on EKF framework, initialization of the EKF and the computational complexity of the resulting algorithms. In Section VI, we present the numerical simulations and the reconstructed pharmacokinetic-rate images using the in vivo breast data. Finally, Section VII summarizes our results.

\section{Spatially Resolved Compartmental Modeling and THE TWO-COMPARTMENT MODEL FOR ICG}

\section{A. Spatially Resolved Compartmental Modeling}

In general, a compartmental model is given by time-dependent coupled ODEs and a measurement model [2]-[4]. Such a model can be extended to include spatial variations in a straightforward manner.

Let $\Omega \subset \mathbb{R}^{3}$ denote the domain of interest. For an $n$-compartment model, let $\mathcal{C}(\boldsymbol{r}, t) \in \mathbb{R}^{n}$ represent the concentration vector in different compartments at location $\boldsymbol{r} \in \Omega$, and at time $t \in\left[T_{0}, T_{1}\right]$; and let $\boldsymbol{\alpha}_{n}(\boldsymbol{r})$ denote the parameter vector whose elements are the pharmacokinetic rates and volume fractions at location $\boldsymbol{r}$. Then, a spatially-resolved compartmental model can be expressed as the following state-space model:

$$
\begin{aligned}
\dot{\mathcal{C}}(\boldsymbol{r}, t) & =\mathcal{K}\left(\boldsymbol{\alpha}_{n}(\boldsymbol{r})\right) \mathcal{C}(\boldsymbol{r}, t) \\
a(\boldsymbol{r}, t) & =\mathcal{V}\left(\boldsymbol{\alpha}_{n}(\boldsymbol{r})\right) \mathcal{C}(\boldsymbol{r}, t) \quad t \in\left[T_{0}, T_{1}\right], \quad \boldsymbol{r} \in \Omega
\end{aligned}
$$

where $\dot{\mathcal{C}}$ denotes the elementwise time-derivative of $\mathcal{C}$, $\mathcal{K}\left(\boldsymbol{r}, \boldsymbol{\alpha}_{n}\right) \in \mathbb{R}^{n \times n}$ is the system matrix whose entries are the pharmacokinetic rates, $a(\boldsymbol{r}, t)$ is the total fluorophore concentration at location $\boldsymbol{r}$ and time $t$, and $\mathcal{V}\left(\boldsymbol{r}, \boldsymbol{\alpha}_{n}\right) \in \mathbb{R}^{n}$ is the vector comprised of volume fractions.

Although our study is applicable to pharmacokinetic modeling of any optical fluorophore, in the following subsection, we will specifically discuss the spatially-resolved pharmacokinetic modeling of ICG using a two-compartment model due to its relevance to breast cancer studies.

\section{B. Two-Compartment Model for the ICG Pharmacokinetics}

ICG is an optical dye commonly used in retinopathy and hepatic diagnostics. Given its low toxicity and FDA approval, it

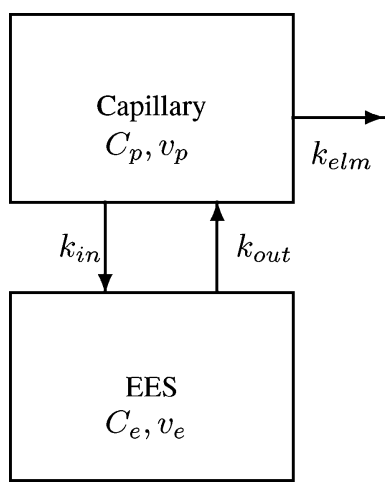

Fig. 1. Block diagram of the two-compartment model for the ICG pharmacokinetics.

has recently been utilized as a blood pooling agent for the detection and diagnosis of cancerous tumors in conjunction with NIR optical methods [14], [15], [17], [21]. In normal tissue, ICG acts as a blood flow indicator in tight capillaries of normal vessels. However in tumors, ICG may act as a diffusible (extravascular) flow in the leaky capillary of cancer vessels. Therefore, the pharmacokinetics of ICG can be used for tumor detection, diagnosis, and staging. Fig. 1 shows the two-compartment model for the ICG kinetics. The two compartments are comprised of plasma and extracellular-extravascular space (EES). Spatially resolved ICG transition between plasma and the EES can be modeled using the following coupled ODEs:

$$
\left[\begin{array}{c}
\dot{C}_{e}(\boldsymbol{r}, t) \\
\dot{C}_{p}(\boldsymbol{r}, t)
\end{array}\right]=\left[\begin{array}{cc}
-k_{\text {out }}(\boldsymbol{r}) & k_{\text {in }}(\boldsymbol{r}) \\
k_{\text {out }}(\boldsymbol{r}) & -\left(k_{\text {in }}+k_{\text {elm }}\right)(\boldsymbol{r})
\end{array}\right]\left[\begin{array}{c}
C_{e}(\boldsymbol{r}, t) \\
C_{p}(\boldsymbol{r}, t)
\end{array}\right]
$$

where $t \in\left[T_{0}, T_{1}\right], \boldsymbol{r} \in \Omega, k_{\text {in }}(\boldsymbol{r})$, and $k_{\text {out }}(\boldsymbol{r})$ are the spatially-resolved pharmacokinetic rates that govern the leakage into and the drainage out of the EES, $k_{\mathrm{elm}}(\boldsymbol{r})$ describes the ICG elimination from the body through kidneys and liver. The vector $\mathcal{C}(\boldsymbol{r}, t)$ in (1) and (2) is composed of $C_{p}(\boldsymbol{r}, t)$, and $C_{e}(\boldsymbol{r}, t)$, representing the ICG concentration in plasma and the EES at $\boldsymbol{r} \in \Omega$ and $t \in\left[T_{0}, T_{1}\right]$, respectively.

The ICG concentration in tissue, $a(\boldsymbol{r}, t)$, is given as a linear combination of the ICG concentration in plasma and the EES

$$
a(\boldsymbol{r}, t)=v_{p}(\boldsymbol{r}) C_{p}(\boldsymbol{r}, t)+v_{e}(\boldsymbol{r}) C_{e}(\boldsymbol{r}, t) \quad t \in\left[T_{0}, T_{1}\right], \quad \boldsymbol{r} \in \Omega
$$

where $v_{p}(\boldsymbol{r}), v_{e}(\boldsymbol{r})$ are spatially-resolved plasma and EES volume fractions, respectively. Here, the unknowns are concentrations in different compartments, pharmacokinetic rates, and volume fractions.

We combine all the pharmacokinetic parameters into a single vector and define

$$
\boldsymbol{\alpha}_{2}(\boldsymbol{r})=\left[\begin{array}{lllll}
k_{\text {in }}(\boldsymbol{r}) & k_{\text {out }}(\boldsymbol{r}) & k_{\mathrm{elm}}(\boldsymbol{r}) & v_{p}(\boldsymbol{r}) & v_{e}(\boldsymbol{r})
\end{array}\right]^{T} .
$$

\section{CONCENTRATION-TO-Measurement MAP FOR FDOT}

In this work, the quantity we wish to reconstruct is the spatially resolved pharmacokinetic-rate images from a sequence 


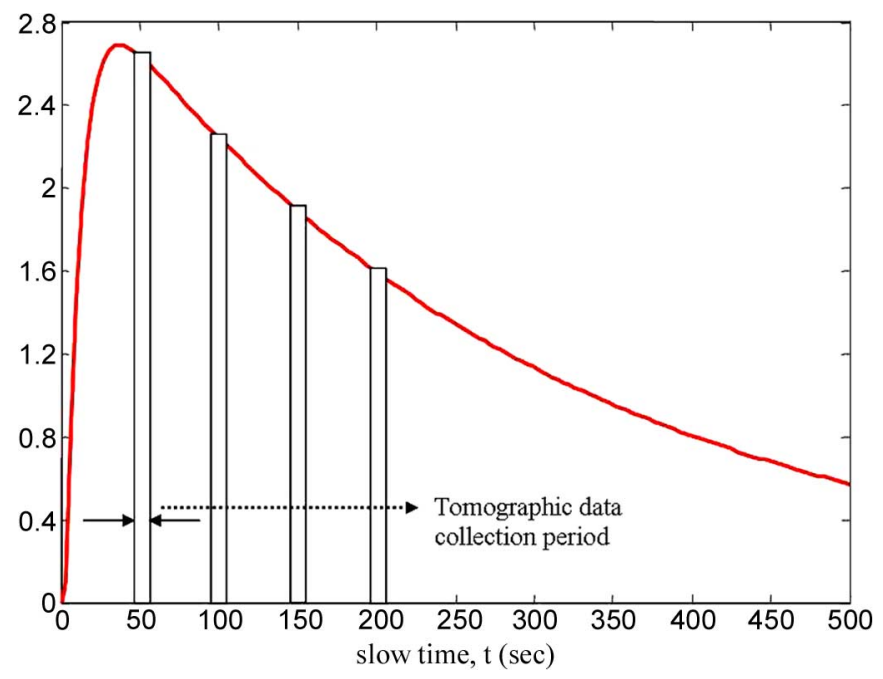

Fig. 2. Total fluorophore concentration versus slow-time variable, $t$.

of boundary measurements obtained by diffuse optical tomographic methods. To do this, we first develop a map which relates sequence of boundary measurements to the spatially resolved fluorophore concentrations. We call this map concentration-to-measurement (CTM) map.

A suitable CTM map can be developed based on a photon propagation model in fluorescing medium. We use diffusion approximation of radiative transfer equation to model photon propagation where the propagation of excitation and emission light are modeled by two coupled diffusion equations.

To relate time-varying fluorophore concentrations to a sequence of boundary measurements, we parameterize time evolution of the fluorophore concentration by a slow-time parameter $t$; and photon propagation during one instance of a tomographic data collection process by a fast-time parameter $t^{\prime}$. Note that $t^{\prime}$ is in the order of the speed of light whereas $t$ is in the order of seconds. Thus, we assume that the absorption and scattering coefficients of tissue are constant during one instance of the tomographic data collection period but vary with the slow-time variable $t$. As a result, frequency domain couple diffusion equation is adequate to model light propagation during the dynamic data collection process. Fig. 2 illustrates a typical time evolution of the fluorophore concentration with respect to slow-time variable $t$.

In the following subsections, for notational brevity, we drop the slow-time dependence in our equations and set $a(\boldsymbol{r}, t)=$ $a(\boldsymbol{r}), \mu_{\mathrm{axf}}(\boldsymbol{r}, t)=\mu_{\mathrm{axf}}(\boldsymbol{r}), \phi_{x, m}(\boldsymbol{r}, \omega, t)=\phi_{x, m}(\boldsymbol{r}, \omega)$, etc. Note that $B_{x, m}$ is a shorthand notation for the quantity $B$ at either excitation or emission wavelengths.

\section{A. Model for Light Propagation in Fluorescing Medium}

The light propagation at the excitation and emission wavelengths can be modelled by the following coupled diffusion equations [30]:

$$
\begin{aligned}
& -\nabla \cdot D_{x}(\boldsymbol{r}) \nabla \phi_{x}(\boldsymbol{r}, \omega)+\left(\mu_{a x}(\boldsymbol{r})+\frac{j \omega}{c}\right) \phi_{x}(\boldsymbol{r}, \omega) \\
& =S(\boldsymbol{r}, \omega), \quad \boldsymbol{r} \in \Omega \subset \mathbb{R}^{3}
\end{aligned}
$$

$$
\begin{aligned}
- & \nabla \cdot D_{m}(\boldsymbol{r}) \nabla \phi_{m}(\boldsymbol{r}, \omega)+\left(\mu_{a m}(\boldsymbol{r})+\frac{j \omega}{c}\right) \phi_{m}(\boldsymbol{r}, \omega) \\
& =-\phi_{x}(\boldsymbol{r}, \omega) \gamma \mu_{\mathrm{axf}}(\boldsymbol{r}) \frac{1-j \omega \tau(\boldsymbol{r})}{1+[\omega \tau(\boldsymbol{r})]^{2}}
\end{aligned}
$$

where the subscripts $x$ and $m$ denote the excitation and emission wavelengths, respectively. $\phi_{x, m}(\boldsymbol{r}, \omega)$ represents the spatially varying optical field in the medium; $\omega$ denotes the modulation frequency of the source (the Fourier transform with respect to the fast-time variable $\left.t^{\prime}\right) ; c$ is the speed of light inside the medium $\Omega ; \tau(\boldsymbol{r})$ is the fluorophore lifetime; $\gamma$ is the fluorophore's quantum efficiency; $\mu_{a x, m}(\boldsymbol{r})$ stands for the spatially varying absorption coefficient of the medium at the excitation and emission wavelengths, respectively, $\mu_{\mathrm{axf}}(\boldsymbol{r})$ is the fluorophore's absorption coefficient; and $\gamma \mu_{\mathrm{axf}}(\boldsymbol{r})$ is the fluorophore yield; $S(\boldsymbol{r}, \omega)$ is the excitation source, $D_{x, m}(\boldsymbol{r})$ is the spatially varying diffusion coefficient given by $D_{x, m}(\boldsymbol{r})=$ $1 / 3\left[\mu_{\boldsymbol{s} x, m}^{\prime}(\boldsymbol{r})+\mu_{a x, m}(\boldsymbol{r})\right]$, where $\mu_{\boldsymbol{s} x, m}^{\prime}(\boldsymbol{r})$ is the reduced scattering coefficient.

The optical coefficients at the excitation and emission wavelengths are due to both the endogenous chromophores and exogenous fluorophore. Thus

$$
\begin{aligned}
\mu_{a x}(\boldsymbol{r}) & =\mu_{\mathrm{axe}}(\boldsymbol{r})+\mu_{\mathrm{axf}}(\boldsymbol{r}) \\
\mu_{\mathrm{am}}(\boldsymbol{r}) & =\mu_{\mathrm{ame}}(\boldsymbol{r})+\mu_{\mathrm{amf}}(\boldsymbol{r}) .
\end{aligned}
$$

Here, the subscript $e$ denotes the endogenous chromophores, and the subscript $f$ denotes the exogenous fluorophore.

We choose Robin-type boundary conditions given as [31]

$$
\begin{aligned}
2 D_{x}(\boldsymbol{r}) \frac{\partial \phi_{x}(\boldsymbol{r}, \omega)}{\partial \nu}+\rho \phi_{x}(\boldsymbol{r}, \omega) & =0, \quad \boldsymbol{r} \in \partial \Omega \\
2 D_{m}(\boldsymbol{r}) \frac{\partial \phi_{m}(\boldsymbol{r}, \omega)}{\partial \nu}+\rho \phi_{m}(\boldsymbol{r}, \omega) & =0
\end{aligned}
$$

where $\partial \Omega$ denotes the boundary of $\Omega, \nu$ denotes the outward normal of the boundary $\partial \Omega, \rho$ is a constant accounting for the refractive index mismatch between the two regions separated by $\partial \Omega$.

Let $a(\boldsymbol{r})$ denote the concentration of fluorophores at $\boldsymbol{r} \in \Omega$. The relationship between $a(\boldsymbol{r})$ and the fluorophore absorption coefficient, $\mu_{a(x, m) f}$, is given by [32]

$$
\mu_{a(x, m) f}(\boldsymbol{r})=\ln 10 \epsilon_{x, m} a(\boldsymbol{r}) \quad \boldsymbol{r} \in \Omega
$$

where $\epsilon_{x, m}$ denotes the fluorophore extinction coefficients at the excitation and emission wavelengths, respectively.

\section{B. Nonlinear Concentration-to-Measurement Map}

Let $\Phi_{m}\left(\boldsymbol{r}_{d}, \boldsymbol{r}_{s} ; \omega\right)$ denote the ratio of the emission data to the excitation data (normalized Born data [31]) at the emission wavelength at the detector location $r_{d}$ due to an excitation source at $\boldsymbol{r}_{s}$. The relationship between $\Phi_{m}\left(\boldsymbol{r}_{d}, \boldsymbol{r}_{s} ; \omega\right)$ and $a(\boldsymbol{r})$ is given by

$$
\begin{aligned}
\Phi_{m}\left(\boldsymbol{r}_{d}, \boldsymbol{r}_{s} ; \omega\right)= & \frac{\ln 10 \epsilon_{x} \gamma}{\phi_{x}\left(\boldsymbol{r}_{d}, \boldsymbol{r}_{s} ; \omega\right)} \\
& \times \int_{\Omega} G_{m}\left(\boldsymbol{r}_{d}, \boldsymbol{r} ; \omega\right) a(\boldsymbol{r}) \frac{1-j \omega \tau(\boldsymbol{r})}{1+[\omega \tau(\boldsymbol{r})]^{2}} \\
& \times \phi_{x}\left(\boldsymbol{r}, \boldsymbol{r}_{s} ; \omega\right) d^{3} \boldsymbol{r} \\
=: & \mathcal{F}_{\boldsymbol{r}_{d}, \boldsymbol{r}_{s}}(a)
\end{aligned}
$$


where $\phi_{x}\left(\boldsymbol{r}, \boldsymbol{r}_{s} ; \omega\right)$ is the photon density at location $\boldsymbol{r}$ due to a source at $\boldsymbol{r}_{s}$ at the excitation wavelength; $G_{m}\left(\boldsymbol{r}_{d}, \boldsymbol{r} ; \omega\right)$ is the Green's function of (7) and (11) at $\boldsymbol{r}_{d}$, due to a point source at $\boldsymbol{r}$, and $\mathcal{F}_{\boldsymbol{r}_{d}, \boldsymbol{r}_{s}}(a)$ is the nonlinear operator that maps the total fluorophore concentration $a$ to the normalized measurements $\Phi_{m}$. Note that the non-linearity in (13) is due to the $\mu_{\text {amf }}$ dependence of $G_{m}\left(\boldsymbol{r}_{d}, \boldsymbol{r} ; \omega\right)$, and $\mu_{a(m, x)}$ dependence of $\phi_{x}$.

We assume that 1) The endogenous absorption coefficients at the emission and the excitation wavelengths are approximately equal, i.e., $\mu_{\text {ame }} \approx \mu_{\text {axe }}$. This is a valid assumption for a number of different applications such as small animal and breast imaging [33]. 2) The diffusion coefficients at both the excitation and emission wavelengths are independent of the endogenous and exogenous absorption coefficients, i.e. $D_{x, m}(\boldsymbol{r}) \approx 1 /\left(3 \mu_{s x, m}^{\prime}(\boldsymbol{r})\right)$. Furthermore, the diffusion coefficients are known but can be spatially varying. 3$)$ The lifetime parameter, $\tau(\boldsymbol{r}), \boldsymbol{r} \in \Omega$, is known, and not necessarily constant.

Let $N_{d}$ and $N_{s}$ denote the number of detectors and sources, respectively. Let $\boldsymbol{\Phi}$ be the measurement vector formed by concatenating the measurements for each source-detector pair as follows:

$$
\boldsymbol{\Phi}=\left[\Phi_{m}\left(\boldsymbol{r}_{d_{1}}, \boldsymbol{r}_{s_{1}} ; \omega\right), \cdots, \Phi_{m}\left(\boldsymbol{r}_{d_{N d}}, \boldsymbol{r}_{s_{N s}} ; \omega\right)\right]^{T} .
$$

Using (13) and (14), we form the following relationship between $a$ and $\boldsymbol{\Phi}$ :

$$
\Phi=: \mathcal{F}(a)
$$

where $\mathcal{F}$ is an operator with matrix kernel in which $\mathcal{F}_{i j}=$ $\mathcal{F}_{\boldsymbol{r}_{d_{i}}, \boldsymbol{r}_{s_{j}}}$ for $i=1, \cdots, N_{d}, j=1, \cdots, N_{s}$.

Note that for notational brevity, we assume a single-frequency measurement model. A multifrequency measurement model is a straightforward extension of the single-frequency measurement model.

\section{Linear Concentration-to-Measurement Map}

An approximate linear map between the normalized measurements and the total fluorophore concentration can be obtained based on the assumption that the presence of exogenous fluorophores does not change the optical coefficients $\mu_{a x, m}$ and $D_{x, m}$ [30]. This assumption leads to the following relationship between the measurements and the fluorophore concentrations:

$$
\begin{aligned}
\Phi_{m}\left(\boldsymbol{r}_{d}, \boldsymbol{r}_{s} ; \omega\right)= & \frac{\ln 10 \epsilon_{x} \gamma}{\phi_{x}^{e}\left(\boldsymbol{r}_{d}, \boldsymbol{r}_{s} ; \omega\right)} \\
& \times \int_{\Omega} G_{m}^{e}\left(\boldsymbol{r}_{d}, \boldsymbol{r} ; \omega\right) a(\boldsymbol{r}) \frac{1-j \omega \tau(\boldsymbol{r})}{1+[\omega \tau(\boldsymbol{r})]^{2}} \\
& \times \phi_{x}^{e}\left(\boldsymbol{r}, \boldsymbol{r}_{s} ; \omega\right) d^{3} \boldsymbol{r} \\
= & : \mathcal{W}_{\boldsymbol{r}_{d}, \boldsymbol{r}_{s}}(a)
\end{aligned}
$$

where $G_{m}^{e}\left(\boldsymbol{r}_{d}, \boldsymbol{r} ; \omega\right)$ is the Green's function of (7) and (11) when $\mu_{a m}=\mu_{\text {ame }}, \phi_{x}^{e}\left(\boldsymbol{r}, \boldsymbol{r}_{s} ; \omega\right)$ is the predicted optical field at $\boldsymbol{r}$ due to a source located at $\boldsymbol{r}_{s}$ when $\mu_{a x}=\mu_{\text {axe }}$, and $\mathcal{W}_{\boldsymbol{r}_{d}, \boldsymbol{r}_{s}}(a)$ denotes the linear operator that maps the normalized measurement at $\boldsymbol{r}_{d}$ due to a source at $\boldsymbol{r}_{s}$ to the total fluorophore concentration.

Forming a measurement vector as in (14), we write

$$
\Phi=: \mathcal{W}(a)
$$

where $\mathcal{W}$ is the linear operator with a matrix kernel in which $\mathcal{W}_{i j}=\mathcal{W}_{\boldsymbol{r}_{d_{i}}, \boldsymbol{r}_{s_{j}}}$ for $i=1, \cdots, N_{d} j=1, \cdots, N_{s}$.

Note that the reconstruction of the pharmacokinetic-rate images that will be discussed in the subsequent sections is not tied to any specific linearization method. Alternatively, a different linear approximation to $\mathcal{F}$ can be obtained by computing its first-order Fréchet derivative to yield a relationship between measurements and total fluorophore concentration. However, we adopted the model in (17) for image reconstruction using in vivo breast data.

\section{Pharmacokinetic-Rates-To-Measurement MaP}

In this section, we combine the CTM map with the spatially resolved compartmental model to obtain a mapping between the spatially resolved pharmacokinetic rates and sequence of boundary measurements. We call this composite map the pharmacokinetic-rate-to-measurement (PTM) map.

Recall that

$$
\dot{\mathcal{C}}(\boldsymbol{r}, t)=\mathcal{K}\left(\boldsymbol{\alpha}_{n}(\boldsymbol{r})\right) \mathcal{C}(\boldsymbol{r}, t) \quad t \in\left[T_{0}, T_{1}\right], \quad \boldsymbol{r} \in \Omega .
$$

Let $\boldsymbol{\Phi}(t)$ denote the measurement vector (16) and $a(\boldsymbol{r}, t)$ denote the flourophore concentration at slow-time parameter $t$. Combining the CTM map (15) with the compartmental model (2), we obtain the following nonlinear relationship:

$$
\boldsymbol{\Phi}(t)=\mathcal{F}\left(\mathcal{V}\left(\boldsymbol{\alpha}_{n}(\boldsymbol{r})\right) \mathcal{C}(\boldsymbol{r}, t)\right), \quad t \in\left[T_{0}, T_{1}\right], \quad \boldsymbol{r} \in \Omega .
$$

For the linear CTM map, (19) becomes

$$
\boldsymbol{\Phi}(t)=\mathcal{W}\left(\mathcal{V}\left(\boldsymbol{\alpha}_{n}(\boldsymbol{r})\right) \mathcal{C}(\boldsymbol{r}, t)\right) \quad t \in\left[T_{0}, T_{1}\right], \quad \boldsymbol{r} \in \Omega .
$$

The (18) and (19), and (18) and (20) constitute the PTM map. We next discretize these equations and incorporate dynamic model uncertainties and measurement noise to the PTM map.

We use first-order Lagrange basis to discretize the domain of interest $\Omega$ (see Section V-B). Let $\boldsymbol{r}_{j}, j=1, \ldots, N$ be the discrete points representing the spatial location of the voxels in $\Omega$. Let $\mathcal{C}\left(\boldsymbol{r}_{j}, t\right)$ represent the concentration vector at time $t$ in different compartments, and $\boldsymbol{\alpha}_{n}\left(\boldsymbol{r}_{j}\right)$ represent the pharmacokinetic rates and volume fractions at the $j$ th voxel centered at $\boldsymbol{r}_{j}$, $j=1, \ldots, N$. Assuming that the dynamic measurements are collected at time instances, $t=k \Delta, k=1, \ldots, K$, where $\Delta$ is the sampling period, we define $\mathbf{C}\left(\boldsymbol{r}_{j}, k\right)=\mathcal{C}\left(\boldsymbol{r}_{j}, k \Delta\right)$, and express the discrete spatially resolved compartmental model as follows:

$$
\mathbf{C}\left(\boldsymbol{r}_{j}, k+1\right)=\mathbf{K}\left(\boldsymbol{\theta}_{n}\left(\boldsymbol{r}_{j}\right)\right) \mathbf{C}\left(\boldsymbol{r}_{j}, k\right)+\boldsymbol{\xi}\left(\boldsymbol{r}_{j}, k\right)
$$

where $k=1, \ldots K, j=1, \ldots N, \boldsymbol{\xi}\left(\boldsymbol{r}_{j}, k\right)$ is a zero-mean Gaussian process with $E\left[\boldsymbol{\xi}\left(\boldsymbol{r}_{j}, k_{1}\right) \boldsymbol{\xi}\left(\boldsymbol{r}_{i}, k_{2}\right)\right]=\delta\left(\boldsymbol{r}_{j}-\right.$ $\left.\boldsymbol{r}_{i}\right) \delta\left(k_{1}-k_{2}\right) \mathbf{Q}_{n}$, representing the dynamic model uncertainty; $\mathbf{K}\left(\boldsymbol{\theta}_{n}\left(\boldsymbol{r}_{j}\right)\right):=e^{\mathcal{K}\left(\boldsymbol{\alpha}_{n}\left(\boldsymbol{r}_{j}\right)\right) \Delta}$ is the discrete-time system matrix as described in [34] and, $\boldsymbol{\theta}_{n}\left(\boldsymbol{r}_{j}\right)$ represents the discrete-time parameter vector for the pharmacokinetic rates and volume fractions. For a detailed discussion of the discretization procedure and an explicit relationship between the parameters $\boldsymbol{\theta}_{n}\left(\boldsymbol{r}_{j}\right)$ and $\boldsymbol{\alpha}_{n}\left(\boldsymbol{r}_{j}\right)$, see [17] and [34]. 
Let $\boldsymbol{\Psi}(k)=\boldsymbol{\Phi}(k \Delta)$. Replacing $\boldsymbol{\alpha}_{n}\left(\boldsymbol{r}_{j}\right)$ with $\boldsymbol{\theta}_{n}\left(\boldsymbol{r}_{j}\right),(19)$ and (20) are discretized as

$$
\begin{aligned}
& \boldsymbol{\Psi}(k)=\mathbf{F}\left(\mathbf{V}\left(\boldsymbol{\theta}_{n}\left(\boldsymbol{r}_{j}\right)\right) \mathbf{C}\left(\boldsymbol{r}_{j}, k\right)\right)+\boldsymbol{\eta}(k) \\
& \boldsymbol{\Psi}(k)=\mathbf{W} \mathbf{V}\left(\boldsymbol{\theta}_{n}\left(\boldsymbol{r}_{j}\right)\right) \mathbf{C}\left(\boldsymbol{r}_{j}, k\right)+\boldsymbol{\eta}(k)
\end{aligned}
$$

where $k=1, \ldots K, j=1, \ldots N, \mathbf{F}$ and $\mathbf{W}$ are the resulting operators when $\boldsymbol{\alpha}_{n}\left(\boldsymbol{r}_{j}\right)$ is replaced with $\boldsymbol{\theta}_{n}\left(\boldsymbol{r}_{j}\right)$ in (13) and (16), respectively; $\boldsymbol{\eta}(k)$ is a zero-mean Gaussian process with $E\left[\boldsymbol{\eta}\left(k_{1}\right) \boldsymbol{\eta}\left(k_{2}\right)\right]=\delta\left(k_{1}-k_{2}\right) \mathbf{R}$ representing the measurement noise, and $\mathbf{V}\left(\boldsymbol{\theta}_{n}\left(\boldsymbol{r}_{j}\right)\right)$ is the vector of discrete volume fractions at $\boldsymbol{r}_{j}$.

For the two-compartment ICG pharmacokinetic model combined with the linear CTM map, the explicit form of (21) and (23) are given by

$$
\begin{aligned}
{\left[\begin{array}{l}
C_{e}\left(\boldsymbol{r}_{j}, k+1\right) \\
C_{p}\left(\boldsymbol{r}_{j}, k+1\right)
\end{array}\right]=} & {\left[\begin{array}{ll}
\tau_{11}\left(\boldsymbol{r}_{j}\right) & \tau_{12}\left(\boldsymbol{r}_{j}\right) \\
\tau_{21}\left(\boldsymbol{r}_{j}\right) & \tau_{22}\left(\boldsymbol{r}_{j}\right)
\end{array}\right]\left[\begin{array}{l}
C_{e}\left(\boldsymbol{r}_{j}, k\right) \\
C_{p}\left(\boldsymbol{r}_{j}, k\right)
\end{array}\right] } \\
& +\left[\begin{array}{l}
\xi_{e}\left(\boldsymbol{r}_{j}, k\right) \\
\xi_{p}\left(\boldsymbol{r}_{j}, k\right)
\end{array}\right] \\
\boldsymbol{\Psi}(k)= & \mathbf{W}\left[v_{e}\left(\boldsymbol{r}_{j}\right)\right. \\
& \left.v_{p}\left(\boldsymbol{r}_{j}\right)\right]\left[\begin{array}{l}
C_{e}\left(\boldsymbol{r}_{j}, k\right) \\
C_{p}\left(\boldsymbol{r}_{j}, k\right)
\end{array}\right] \\
& +\boldsymbol{\eta}(k)
\end{aligned}
$$

where

$$
\begin{aligned}
\mathbf{C}\left(\boldsymbol{r}_{j}, k\right) & =\left[\begin{array}{ll}
C_{e}\left(\boldsymbol{r}_{j}, k\right) & C_{p}\left(\boldsymbol{r}_{j}, k\right)
\end{array}\right]^{T} \\
\boldsymbol{\xi}\left(\boldsymbol{r}_{j}, k\right) & =\left[\begin{array}{ll}
\xi_{e}\left(\boldsymbol{r}_{j}, k\right) & \xi_{p}\left(\boldsymbol{r}_{j}, k\right)
\end{array}\right]^{T} \\
\mathbf{V}\left(\boldsymbol{\theta}_{2}\left(\boldsymbol{r}_{j}\right)\right) & =\left[\begin{array}{ll}
v_{e}\left(\boldsymbol{r}_{j}\right) & v_{p}\left(\boldsymbol{r}_{j}\right)
\end{array}\right] \\
\boldsymbol{\theta}_{2}\left(\boldsymbol{r}_{j}\right) & =\left[\begin{array}{lll}
\tau_{11}\left(\boldsymbol{r}_{j}\right) & \tau_{12}\left(\boldsymbol{r}_{j}\right) \tau_{21}\left(\boldsymbol{r}_{j}\right) \tau_{22}\left(\boldsymbol{r}_{j}\right) v_{e}\left(\boldsymbol{r}_{j}\right) v_{p}\left(\boldsymbol{r}_{j}\right)
\end{array}\right]^{T} .
\end{aligned}
$$

Here, $\mathbf{K}\left(\boldsymbol{\theta}_{n}\left(\boldsymbol{r}_{j}\right)\right)$ is the $2 \times 2$ system matrix in (24), for $j=$ $1, \ldots, N$ and $k=1, \ldots, K$.

\section{ReCONSTRUCTION OF PHARMACOKINETIC-RATE AND CONCENTRATION IMAGES FROM THE BOUNDARY MEASUREMENTS}

The forward model in (21) and (22), (23) forms a state-space model, (21) being the state equation and (22), (23) being the measurement equation. In this section, we discuss the estimation of the system parameters, $\boldsymbol{\theta}_{n}\left(\boldsymbol{r}_{j}\right)$, and the states $\mathbf{C}_{d}\left(\boldsymbol{r}_{j}, k\right)$ for $j=1, \ldots, N$, from the measurements $\boldsymbol{\Psi}(k), k=1, \ldots, K$ using the extended Kalman filtering (EKF) framework. For the advantages of EKF framework see Section I.

Note that both the fluorophore concentrations in different compartments, $\mathbf{C}\left(\boldsymbol{r}_{j}, k\right)$, and the system parameters, $\boldsymbol{\theta}_{n}\left(\boldsymbol{r}_{j}\right)$, are unknown. In this case, we estimate both the states and system parameters from measurements within the EKF framework. To do so, we regard the state equation in (21) as a nonlinear equation in which the system parameters and states are combined to form the new states of the nonlinear equation. We then iteratively linearize the nonlinear state equation and solve for the new unknown states using the EKF framework. This approach requires use of temporal prior models on $\boldsymbol{\theta}_{n}\left(\boldsymbol{r}_{j}\right)$. We describe one such model in the following subsection.

\section{A. A Priori Model for Pharmacokinetic Rates and Volume Fractions}

To impose a temporal prior model on $\boldsymbol{\theta}_{n}\left(\boldsymbol{r}_{j}\right)$, we extend our notation to $\boldsymbol{\theta}_{n}\left(\boldsymbol{r}_{j}, k\right), k=1, \ldots, K$. Note that $\boldsymbol{\theta}_{n}\left(\boldsymbol{r}_{j}, k\right)$ is a vector containing pharmacokinetic rates and volume fractions at location $\boldsymbol{r}_{j} \in \Omega$ and time $k \Delta$. For each element, $\tilde{\theta_{n}}\left(\boldsymbol{r}_{j}, k\right)$, of the vector $\boldsymbol{\theta}_{n}\left(\boldsymbol{r}_{j}, k\right)$, we impose the following dynamic model:

$$
\tilde{\theta_{n}}\left(\boldsymbol{r}_{j}, k+1\right)=\tilde{\theta_{n}}\left(\boldsymbol{r}_{j}, k\right)+\tilde{\varsigma_{1}}\left(\boldsymbol{r}_{j}, k\right)
$$

where $\tilde{\varsigma}_{1}\left(\boldsymbol{r}_{j}, k\right)$ is a zero-mean Gaussian process with $E\left[\tilde{S}_{1}\left(\boldsymbol{r}_{1}, k_{1}\right) \tilde{\varsigma}_{1}\left(\boldsymbol{r}_{2}, k_{2}\right)\right]=\delta\left(\boldsymbol{r}_{1}-\boldsymbol{r}_{2}\right) \delta\left(k_{1}-k_{2}\right) z_{1}, z_{1}>0$.

Note that $\boldsymbol{\theta}_{n}\left(\boldsymbol{r}_{j}\right)$ is modeled as a time-independent parameter, and the model in (26) relates $\tilde{\theta_{n}}\left(\boldsymbol{r}_{j}, k+1\right)$ and $\tilde{\theta_{n}}\left(\boldsymbol{r}_{j}, k\right)$ with an all-pass filter. If, on the other hand, $\boldsymbol{\theta}_{n}\left(\boldsymbol{r}_{j}\right)$ is time-dependent, a different filter can be chosen based on a priori physiological information and/or robustness considerations.

In addition to the temporal prior, we impose a spatial smoothing prior on $\boldsymbol{\theta}_{n}\left(\boldsymbol{r}_{j}, k\right)$ to improve the robustness of the reconstruction with respect to measurement noise and to incorporate a priori physiological information into image reconstruction. This model is given as

$$
\tilde{\theta_{n}}\left(\boldsymbol{r}_{j}, k\right)=\sum_{l=1\left(j_{l} \neq j\right)}^{M} \tilde{\beta}_{l} \tilde{\theta}_{n}\left(\boldsymbol{r}_{j_{l}}, k\right)+\tilde{\varsigma_{2}}\left(\boldsymbol{r}_{j}, k\right)
$$

where $j_{l}, l=1, \ldots, M\left(j_{l} \neq j\right)$ are the indices of the voxels in the neighborhood of the $j$ th voxel; $\tilde{\beta}_{l}, l=1, \ldots, M$ are the spatial weighting coefficients, which may be different for each pharmacokinetic-rate or volume fraction image; and $\tilde{\varsigma}_{2}\left(\boldsymbol{r}_{j}, k\right)$, is a zero-mean Gaussian process with $E\left[\tilde{\varsigma}_{2}\left(\boldsymbol{r}_{1}, k_{1}\right) \tilde{\varsigma}_{2}\left(\boldsymbol{r}_{2}, k_{2}\right)\right]=$ $\delta\left(\boldsymbol{r}_{1}-\boldsymbol{r}_{2}\right) \delta\left(k_{1}-k_{2}\right) z_{2}, z_{2}>0$.

The weighting coefficients $\tilde{\beta}_{l}$ may be spatially varying and can be chosen based on a variety of physiological information, i.e., tumor location, size, or shape. In our numerical simulations and in vivo data processing, we assumed that no such specific information about the tumor is available and used equal weights, i.e., $\tilde{\beta}_{l}=1 / M$. This choice imposes an isotropic smoothing on the $\tilde{\theta}_{n}\left(\boldsymbol{r}_{j}, k\right)$ estimates.

Inserting the right-hand side of (27) for $\tilde{\theta}_{n}\left(\boldsymbol{r}_{j}, k\right)$ in (26), we obtain the following spatio-temporal model for each entry $\tilde{\theta_{n}}\left(\boldsymbol{r}_{j}, k\right)$ of the vector $\boldsymbol{\theta}_{n}\left(\boldsymbol{r}_{j}, k\right)$ :

$$
\tilde{\theta_{n}}\left(\boldsymbol{r}_{j}, k+1\right)=\sum_{l=1\left(j_{l} \neq j\right)}^{M} \tilde{\beta}_{l} \tilde{\theta_{n}}\left(\boldsymbol{r}_{j_{l}}, k\right)+\tilde{\varsigma}\left(\boldsymbol{r}_{j}, k\right)
$$

where $\tilde{\varsigma}\left(\boldsymbol{r}_{j}, k\right)$ is a zero-mean Gaussian process with $E\left[\tilde{\varsigma}\left(\boldsymbol{r}_{1}, k_{1}\right) \tilde{\varsigma}\left(\boldsymbol{r}_{2}, k_{2}\right)\right]=\delta\left(\boldsymbol{r}_{1}-\boldsymbol{r}_{2}\right) \delta\left(k_{1}-k_{2}\right) z, z>0$, and $z$ is a function of $z_{1}$ and $z_{2}$. Fig. 3 illustrates the resulting neighborhood system for 2-D images for $M=4$.

Note that it is possible to develop an alternative spatio-temporal neighborhood system taking into account the 4-D nature of the parameter space.

To simplify our notation, we express (28) in vector notation for all entries of the vector $\boldsymbol{\theta}_{n}\left(\boldsymbol{r}_{j}, k\right)$ as follows:

$$
\boldsymbol{\theta}_{n}\left(\boldsymbol{r}_{j}, k+1\right)=\boldsymbol{\beta}_{j}\left(\boldsymbol{\theta}_{n}\left(\boldsymbol{r}_{j_{1}}, k\right), \ldots, \boldsymbol{\theta}_{n}\left(\boldsymbol{r}_{j_{M}}, k\right)\right)+\boldsymbol{\varsigma}\left(\boldsymbol{r}_{j}, k\right)
$$

where $\boldsymbol{\beta}_{j}$ is a vector-valued linear function of $\boldsymbol{\theta}_{n}\left(\boldsymbol{r}_{j_{l}}, k\right)$, $l=1, \ldots, M$ as defined in (28) and $\boldsymbol{\varsigma}\left(\boldsymbol{r}_{j}, k\right)$ is formed by concatenating the $\tilde{\varsigma}\left(\boldsymbol{r}_{j}, k\right)$ into a column vector. It is a 


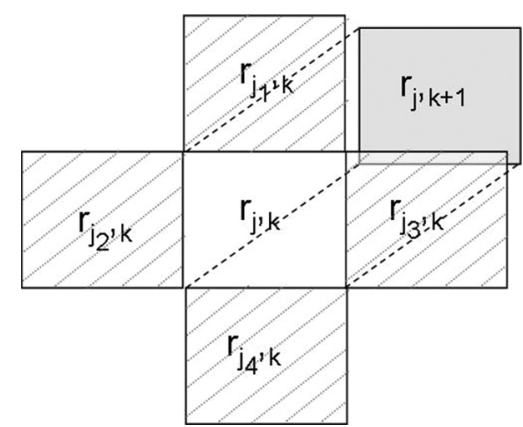

Fig. 3. An illustration of the spatio-temporal neighborhood system for 2-D pharmacokinetic-rate and volume fraction images for $M=4$. Based on the model in (28)-(30) the neighborhood of the voxel at $r_{j, k+1}$ (gray square) is given by the voxels at $r_{j_{1}, k}, r_{j_{2}, k}, r_{j_{3}, k}$ and $r_{j_{4}, k}$ denoted by gray-dashed squares.

zero-mean Gaussian process with $E\left[\boldsymbol{\varsigma}\left(\boldsymbol{r}_{1}, k_{1}\right) \boldsymbol{\varsigma}\left(\boldsymbol{r}_{2}, k_{2}\right)\right]=$ $\delta\left(\boldsymbol{r}_{1}-\boldsymbol{r}_{2}\right) \delta\left(k_{1}-k_{2}\right) \mathbf{Z}_{1}$.

\section{B. Estimation of Pharmacokinetic-Rate Images by Extended Kalman Filterin}

Our objective is to estimate the fluorophore concentration images in different compartments, and pharmacokinetic-rate images. To do so, we first concatenate the concentration vectors $\mathbf{C}\left(\boldsymbol{r}_{j}, k\right)$ and the parameter vectors $\boldsymbol{\theta}_{n}\left(\boldsymbol{r}_{j}, k\right)$ for all voxels, $j=1, \ldots, N$ and form the following vectors:

$$
\begin{aligned}
\mathbf{C}(k) & =\left[\begin{array}{lll}
\mathbf{C}^{T}\left(\boldsymbol{r}_{1}, k\right) & \ldots & \mathbf{C}^{T}\left(\boldsymbol{r}_{N}, k\right)
\end{array}\right]^{T} \\
\boldsymbol{\theta}_{n}(k) & =\left[\begin{array}{lll}
\boldsymbol{\theta}_{n}^{T}\left(\boldsymbol{r}_{1}, k\right) & \ldots & \boldsymbol{\theta}_{n}^{T}\left(\boldsymbol{r}_{N}, k\right)
\end{array}\right]^{T} .
\end{aligned}
$$

Next, we concatenate the vectors $\mathbf{C}(k)$ and $\boldsymbol{\theta}_{n}(k)$ and form the new state-space model based on (21) and (29)

$$
\begin{aligned}
& {\left[\begin{array}{c}
\mathbf{C}(k+1) \\
\boldsymbol{\theta}_{n}(k+1)
\end{array}\right]=\left[\begin{array}{c}
\mathbf{K}\left(\boldsymbol{\theta}_{n}(k)\right) \mathbf{C}(k) \\
\boldsymbol{\beta}\left(\boldsymbol{\theta}_{n}(k)\right)
\end{array}\right]+\left[\begin{array}{l}
\boldsymbol{\xi}(k) \\
\boldsymbol{\varsigma}(k)
\end{array}\right]} \\
& \boldsymbol{\Psi}(k)=\left[\mathbf{F}\left(\mathbf{V}\left(\boldsymbol{\theta}_{n}(k)\right) \mathbf{C}(k)\right) \quad 0\right]\left[\begin{array}{c}
1 \\
\boldsymbol{\theta}_{n}(k)
\end{array}\right] \\
& +\boldsymbol{\eta}(k) \\
& \boldsymbol{\Psi}(k)=\left[\ln 10 \epsilon_{x} \mathbf{W V}\left(\boldsymbol{\theta}_{n}(k)\right) \mathbf{C}(k) \quad 0\right]\left[\begin{array}{c}
1 \\
\boldsymbol{\theta}_{n}(k)
\end{array}\right] \\
& +\boldsymbol{\eta}(k),
\end{aligned}
$$

where

$$
\begin{aligned}
& \mathbf{K}\left(\boldsymbol{\theta}_{n}(k)\right) \mathbf{C}(k)=\left[\begin{array}{c}
\mathbf{K}\left(\boldsymbol{\theta}_{n}\left(\boldsymbol{r}_{1}, k\right)\right) \mathbf{C}\left(\boldsymbol{r}_{1}, k\right) \\
\vdots \\
\mathbf{K}\left(\boldsymbol{\theta}_{n}\left(\boldsymbol{r}_{N}, k\right)\right) \mathbf{C}\left(\boldsymbol{r}_{N}, k\right)
\end{array}\right] \\
& \boldsymbol{\beta}\left(\boldsymbol{\theta}_{n}(k)\right)=\left[\begin{array}{c}
\boldsymbol{\beta}_{1}\left(\boldsymbol{\theta}_{n}\left(\boldsymbol{r}_{1}, k\right), \ldots\right) \\
\vdots \\
\boldsymbol{\beta}_{N}\left(\boldsymbol{\theta}_{n}\left(\boldsymbol{r}_{N}, k\right), \ldots\right)
\end{array}\right] \\
& \mathbf{F}\left(\mathbf{V}\left(\boldsymbol{\theta}_{n}(k)\right) \mathbf{C}(k)\right)=\left[\begin{array}{c}
\mathbf{F}\left(\mathbf{V}\left(\boldsymbol{\theta}_{n}\left(\boldsymbol{r}_{1}, k\right)\right) \mathbf{C}\left(\boldsymbol{r}_{1}, k\right)\right) \\
\vdots \\
\mathbf{F}\left(\mathbf{V}\left(\boldsymbol{\theta}_{n}\left(\boldsymbol{r}_{N}, k\right)\right) \mathbf{C}\left(\boldsymbol{r}_{N}, k\right)\right)
\end{array}\right]^{T} \\
& \boldsymbol{\xi}(k)=\left[\begin{array}{lll}
\boldsymbol{\xi}\left(\boldsymbol{r}_{1}, k\right) & \ldots & \boldsymbol{\xi}\left(\boldsymbol{r}_{N}, k\right)
\end{array}\right]^{T} \\
& \boldsymbol{\varsigma}(k)=\left[\begin{array}{lll}
\boldsymbol{\zeta}\left(\boldsymbol{r}_{1}, k\right) & \ldots & \boldsymbol{\varsigma}\left(\boldsymbol{r}_{N}, k\right)
\end{array}\right]^{T}
\end{aligned}
$$

where $\boldsymbol{\xi}(k)$ and $\boldsymbol{\varsigma}(k)$ are zero-mean Gaussian processes with covariance matrices $\mathbf{Q}$ and $\mathbf{Z}$, respectively. $\boldsymbol{\eta}(k)$ is the zeromean Gaussian process with covariance matrix $\mathbf{R}$ as defined before.

Note that although $\boldsymbol{\beta}$ is linear in $\boldsymbol{\theta}_{n}\left(\boldsymbol{r}_{j}, k\right), j=1, \ldots, N$, (30) is nonlinear in new states. Furthermore, the state (30) is not block diagonal.

We next utilize the EKF framework to estimate the fluorophore concentration images in different compartments and pharmacokinetic-rate images based on (30)-(33). Table I tabulates the steps of the EKF algorithm. The terms in Table I are defined as follows: $\hat{\mathbf{C}}(k \mid k-1)$ and $\hat{\boldsymbol{\theta}}_{n}(k \mid k-1)$ are the concentration and parameter estimates at time $k$ given all the measurements up to time $k-1$, respectively. Similarly, $\hat{\mathbf{C}}(k)$ and $\hat{\boldsymbol{\theta}}_{n}(k)$ are the concentration and parameter estimate updates at time $k$, respectively. $\mathbf{P}_{k, k-1}$ denotes the error covariance propagation at time $k$ given all the measurements up to time $k-1 ; \mathbf{P}_{k, k}$ is the error covariance update at time $k . \mathbf{H}_{k}$ is the recursive Kalman gain matrix at time $k$ and $\mathbf{I}$ is the identity matrix. $\mathbf{J}_{k-1}$ is the Jacobian matrix due to iterative linearization of the (30) around $\hat{\mathbf{C}}(k-1)$ and $\hat{\boldsymbol{\theta}}_{n}(k-1) . \boldsymbol{\Lambda}_{k \mid k-1}$ is the matrix formed by the discretized Fréchet derivatives of $\mathcal{F}$ with respect to $\mathcal{C}$ and $\boldsymbol{\theta}_{n}$ at the updates $\hat{\mathbf{C}}(k \mid k-1)$ and $\hat{\boldsymbol{\theta}}_{n}(k \mid k-1)$. In Table I, the EKF algorithm is presented for the nonlinear case. For the linear case, the nonlinear operator $\mathbf{F}$ is simply replaced by the linear operator $\mathbf{W}$.

The first-order Fréchet derivative of $\mathcal{F}$ (or $\mathcal{F}_{\boldsymbol{r}_{d}, \boldsymbol{r}_{s}}$ ) with respect to the fluorophore concentration $a$ at the EKF total concentration estimate at the $(k \mid k-1)$ th step is given by [31], [35], [36]

$$
\begin{aligned}
& \partial \mathcal{F}_{\boldsymbol{r}_{d}, \boldsymbol{r}_{s}}(\delta a)= \frac{\ln 10 \epsilon_{x}}{\Phi_{x}^{k \mid k-1}\left(\boldsymbol{r}_{d}, \boldsymbol{r}_{s} ; \omega\right)} \\
& \times\left(\int_{\Omega} G_{m}^{k \mid k-1}\left(\boldsymbol{r}_{d}, \boldsymbol{r} ; \omega\right) \Phi_{x}^{k \mid k-1}\left(\boldsymbol{r}, \boldsymbol{r}_{s} ; \omega\right)\right. \\
& \times \frac{1-j \omega \tau(\boldsymbol{r})}{1+[\omega \tau(\boldsymbol{r})]^{2}} \gamma \delta a(\boldsymbol{r}) d \boldsymbol{r} \\
&-\int_{\Omega} G_{m}^{k \mid k-1}\left(\boldsymbol{r}_{d}, \boldsymbol{r} ; \omega\right) \Phi_{m}^{k \mid k-1}\left(\boldsymbol{r}, \boldsymbol{r}_{s} ; \omega\right) \\
& \times \frac{1-j \omega \tau(\boldsymbol{r})}{1+[\omega \tau(\boldsymbol{r})]^{2}} \frac{\epsilon_{m}}{\epsilon_{x}} \delta a(\boldsymbol{r}) d \boldsymbol{r} \\
&-\int_{\Omega} G_{m x}^{k \mid k-1}\left(\boldsymbol{r}_{d}, \boldsymbol{r} ; \omega\right) \Phi_{x}^{k \mid k-1}\left(\boldsymbol{r}, \boldsymbol{r}_{s} ; \omega\right) \\
&\left.\times \frac{1-j \omega \tau(\boldsymbol{r})}{1+[\omega \tau(\boldsymbol{r})]^{2}} \delta a(\boldsymbol{r}) d \boldsymbol{r}\right)
\end{aligned}
$$

where we define $\Phi_{x}^{k \mid k-1}\left(\boldsymbol{r}, \boldsymbol{r}_{s} ; \omega\right)$ as the solution of (6) and $(10), \Phi_{m}^{k \mid k-1}\left(\boldsymbol{r}, \boldsymbol{r}_{s} ; \omega\right)$ as the solution of (7) and (11), $G_{m}^{k \mid k-1}\left(\boldsymbol{r}_{d}, \boldsymbol{r} ; \omega\right)$ as the Green's function of (7) and (11), and $G_{m x}^{k \mid k-1}\left(\boldsymbol{r}_{d}, \boldsymbol{r} ; \omega\right)$ as the solution of (6) and (10) where $S(\boldsymbol{r}, \omega)$ in (6) is replaced by $\gamma \mu_{\mathrm{axf}} G_{m}^{k \mid k-1}\left(\boldsymbol{r}_{d}, \boldsymbol{r} ; \omega\right)$ given the EKF estimates of the fluorophore concentrations at different compartments at the $(k \mid k-1)$ th step [35]. In (34), the first integral results from the right-hand side of (7), while the second and third integrals originate from the dependence of $\mu_{a m}$ and 
TABLE I

EKF Algorithm For the Simultaneous Estimation of Fluorophore ConCEntration, Pharmacokinetic-Rate, AND Volume Fraction Images

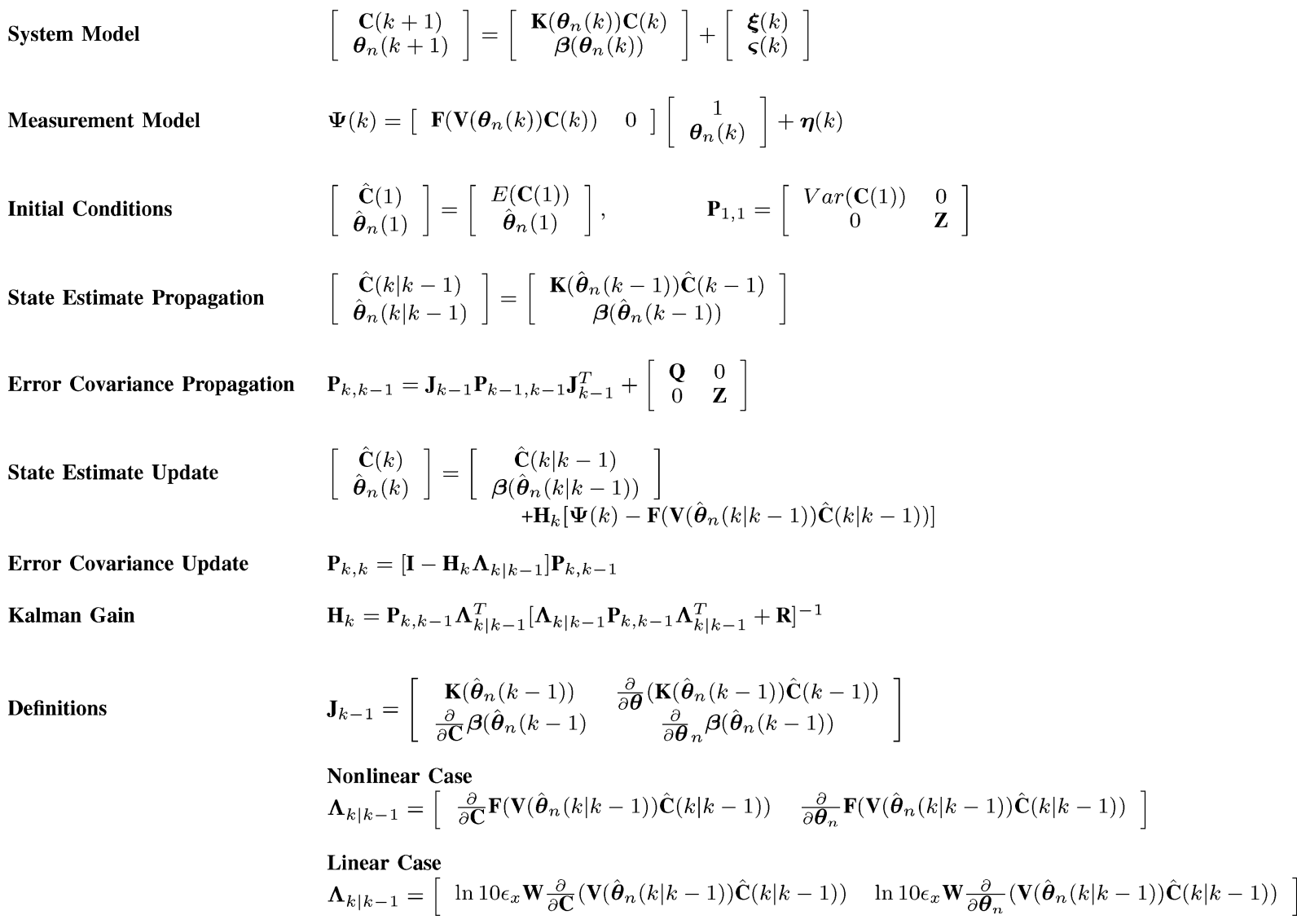

$\mu_{a x}$, respectively, on the unknown fluorophore absorption coefficient. We note that the kernels of the second and thirds integrals are much smaller than the kernel of the first integral. Therefore, the first integral in (34) dominates and the rest can be neglected. As a result, The first-order Fréchet derivative of $\mathcal{F}$ (or $\mathcal{F}_{\boldsymbol{r}_{d}, \boldsymbol{r}_{s}}$ ) with respect to the fluorophore concentration $a$ at the EKF total concentration estimate at the $(k \mid k-1)$ th step can be approximated by

$$
\begin{array}{r}
\partial \mathcal{F}_{\boldsymbol{r}_{d}, \boldsymbol{r}_{s}}(\delta a)=\frac{\ln 10 \gamma \epsilon_{x}}{\Phi_{x}^{k \mid k-1}\left(\boldsymbol{r}_{d}, \boldsymbol{r}_{s} ; \omega\right)} \int_{\Omega} G_{m}^{k \mid k-1}\left(\boldsymbol{r}_{d}, \boldsymbol{r} ; \omega\right) \Phi_{x}^{k \mid k-1} \\
\times\left(\boldsymbol{r}, \boldsymbol{r}_{s} ; \omega\right) \frac{1-j \omega \tau(\boldsymbol{r})}{1+[\omega \tau(\boldsymbol{r})]^{2}} \delta a(\boldsymbol{r}) d \boldsymbol{r} .
\end{array}
$$

Based on the assumptions 1)-3) in Section III-A, the terms in the kernel of (35) can be computed as follows: Based on the assumption $i$ ), $\mu_{\mathrm{axe}} \approx \mu_{\mathrm{ame}}$. Furthermore, $\mu_{\mathrm{amf}}$ and $\mu_{\mathrm{axf}}$ are linearly dependent by (12). Thus, given the $\mu_{\text {axe }}$ reconstruction based on (6) and (10) at the $k$ th step, and $\mu_{\text {amf }}$ obtained via EKF estimates (fluorophore concentrations at different compartments and volume fractions) at the $(k \mid k-1)$ th step, $G_{m}^{k \mid k-1}\left(\boldsymbol{r}_{d}, \boldsymbol{r} ; \omega\right)$ can be computed using (7) and (11). Similarly, given the $\mu_{\text {axe }}$ reconstruction based on (6) and (10) at the $k$ th step, and $\mu_{\mathrm{axf}}$ estimate obtained via the EKF estimates of fluorophore concentrations at different compartments and volume fractions at the $(k \mid k-1)$ th step, $\Phi_{x}^{k \mid k-1}\left(\boldsymbol{r}_{d}, \boldsymbol{r}_{s} ; \omega\right)$ can be computed as the solution of (6) and (10). Finally, we assume that $1-j \omega \tau(\boldsymbol{r}) / 1+$ $[\omega \tau(\boldsymbol{r})]^{2}$ is known.

Note that the calculation of the Fréchet derivative can be simplified under some additional assumptions. If $\mu_{\text {axe }}$ does not vary with the slow-time variable $k$, and that $\mu_{\text {amf }} \ll \mu_{\text {ame, }}$, then $G_{m}\left(\boldsymbol{r}_{d}, \boldsymbol{r} ; \omega\right)$ can be computed with respect to $\mu_{\text {axe }}$ and remains invariant with respect to the slow-time variable $k$. Using the chain rule, the first-order Fréchet derivative of $\mathcal{F}_{\boldsymbol{r}_{d}, \boldsymbol{r}_{s}}$ with respect to each element of $\mathcal{C}$ is given by

$$
\begin{aligned}
\partial \mathcal{F}_{\boldsymbol{r}_{d}, \boldsymbol{r}_{s}}\left(\delta \mathcal{C}_{i}\right)= & \frac{\ln 10 \gamma \epsilon_{x}}{\Phi_{x}^{k \mid k-1}\left(\boldsymbol{r}_{d}, \boldsymbol{r}_{s} ; \omega\right)} \int_{\Omega} G_{m}^{k \mid k-1}\left(\boldsymbol{r}_{d}, \boldsymbol{r} ; \omega\right) \Phi_{x}^{k \mid k-1} \\
& \times\left(\boldsymbol{r}, \boldsymbol{r}_{s} ; \omega\right) \frac{1-j \omega \tau(\boldsymbol{r})}{1+[\omega \tau(\boldsymbol{r})]^{2}} \delta \mathcal{C}_{i} v_{i}(\boldsymbol{r}) d \boldsymbol{r}
\end{aligned}
$$

where $v_{i}, i=1,2, \ldots, n$, denotes the volume fractions.

Similarly, the first-order Fréchet derivative of $\mathcal{F}_{\boldsymbol{r}_{d}, \boldsymbol{r}_{s}}$ with respect to each element of $\boldsymbol{\alpha}_{n}$ is given by

$$
\begin{aligned}
\partial \mathcal{F}_{\boldsymbol{r}_{d}, \boldsymbol{r}_{s}}\left(\delta \boldsymbol{\alpha}_{n}\right)= & \frac{\ln 10 \gamma \epsilon_{x}}{\Phi_{x}^{k \mid k-1}\left(\boldsymbol{r}_{d}, \boldsymbol{r}_{s} ; \omega\right)} \int_{\Omega} G_{m}^{k \mid k-1}\left(\boldsymbol{r}_{d}, \boldsymbol{r} ; \omega\right) \Phi_{x}^{k \mid k-1} \\
& \times\left(\boldsymbol{r}, \boldsymbol{r}_{s} ; \omega\right) \frac{1-j \omega \tau(\boldsymbol{r})}{1+[\omega \tau(\boldsymbol{r})]^{2}} \delta v_{i}(\boldsymbol{r}) \mathcal{C}_{i}(\boldsymbol{r}) d r .
\end{aligned}
$$

Note that $\boldsymbol{\alpha}_{n}$ contains both pharmacokinetic-rate and volume fraction parameters. However, the Fréchet derivative of $\mathcal{F}$ with 
respect to pharmacokinetic rates is zero since $\mathcal{F}$ depends only on the volume fractions.

In our numerical simulations, we used finite elements with piecewise linear first-order Lagrange polynomials to discretize the domain $\Omega$ and thus to discretize the Fréchet derivatives given in (36) and (37).

The EKF algorithms for the estimation of concentration and parameter images are different for the linear and nonlinear measurement models given in (13) and (16). When the linearized measurement model (16) is employed, the operator $\mathcal{W}$ is assumed to be constant throughout the dynamic update of the fluorophore concentration. This leads to the inherent assumption that the dynamic changes in the fluorophore concentration can be modeled as a perturbation on the endogenous chromophore concentrations. For the nonlinear measurement model (13), on the other hand, the Fréchet derivative is updated at every iteration of the EKF algorithm based on the $(k \mid k-1)$ th update of the concentration and volume fraction estimates. While computationally more intense, the EKF algorithm based on the nonlinear measurement model eliminates the limiting assumption of restricting the dynamic changes to perturbations from a constant endogenous chromophore concentration.

\section{Convergence of EKF and Compartmental Model Mismatch}

The convergence of the EKF algorithm is related to the appropriate compartmental model selection. In the context of EKF, compartmental model selection involves compartmental model order selection and the initialization of the covariance matrices $\mathbf{R}, \mathbf{Q}$ and $\mathbf{Z}$. From the biological point of view, the higher the number of compartments, the better the pharmacokinetic model would be. However, ICG being effectively a large molecule, a two-compartment model is assumed to be sufficient to capture its pharmacokinetics [17]-[19]. Furthermore, higher order compartmental modeling involves estimation of larger number of unknown parameters. In [17], we proposed the Bayesian information criterion (BIC), to select the best model order. BIC provides a quantitative model order selection metric representing a compromise between the accuracy of the estimates and the number of unknowns to be estimated given the available data. In this paper, we assume that an appropriate model order is determined a priori based on biological and/or estimation-theoretic criteria for the fluorophore of interest and focus on the convergence of the EKF and the initialization of the covariance matrices $\mathbf{R}, \mathbf{Q}$, and $\mathbf{Z}$.

The convergence properties of the EKF has been well-studied in the literature [28], [37]-[40]. In general, for the joint estimation of parameters and states, the estimates may be biased or divergent.

In [39], it was shown that the main cause of divergence in EKF is due to lack of coupling between the Kalman gain $\mathbf{H}_{k}$ and the discrete parameter vector $\boldsymbol{\theta}_{n}$ and a modification to overcome this issue was described.

However, this modification imposes a high computational load on the resulting algorithm [38], [39]. In [37]-[39], it was shown that the asymptotic convergence rate of EKF depends on the proper selection of the covariance matrices $\mathbf{R}, \mathbf{Q}$, and $\mathbf{Z}$; with $\mathbf{R}$ being the most important term controlling the convergence rate of the EKF [37]. Therefore, in many practical
TABLE II

RANGE OF VALUES FOR THE PARAMETERS USED IN COMPUTATIONAL COMPLEXITY ANALYSIS

\begin{tabular}{ll}
\hline Number of compartments, $n$ & $2-4$ \\
Total number of voxels, $N$ & $576-649$ \\
Size of measurement vector, $m$ & $128-256$ \\
Total number of steps, $K$ & $50-100$ \\
\hline
\end{tabular}

applications, "tuning of the filter" approach, which involves manual adjustments of the covariances matrices $\mathbf{R}, \mathbf{Q}$, and $\mathbf{Z}$, is adopted. In our work, as proposed in [38] and [39], we regard the covariance matrices $\mathbf{R}, \mathbf{Q}$, and $\mathbf{Z}$ as tuning parameters and choose the values that lead to the minimum norm (trace sum) of the error covariance matrix within biological limits. We empirically evaluate the convergence of EKF and the goodness of the compartmental model fit by studying the change in the residuals of measurements with respect to the number of iterations given the covariance matrices $\mathbf{R}, \mathbf{Q}$, and $\mathbf{Z}$ [41]. For numerical results, see Sections VI-A and VI-B.

\section{Computational Complexity of the EKF-Based Reconstruction Algorithms}

In this section, we derive the computational complexity of the EKF-based direct reconstruction algorithms based on the linear and nonlinear models in (31) and (32) under the assumptions outlined in Sections VI-A and VI-B. We next derive the computational complexity of the EKF-based voxel-by-voxel pharmacokinetic-rate and concentration image reconstruction algorithm that we introduced in [21] and compare it with the computational complexity of the algorithms introduced in this paper.

The computational complexity of one recursion of the EKF is $\mathcal{O}\left(2 m^{2} h\right)+\mathcal{O}\left(2 m h^{2}\right)+\mathcal{O}\left(m^{3}\right)+\mathcal{O}\left(h^{3}\right)$, where $m$ denotes the dimension of the measurement vector, and $h$ denotes the dimension of the states [42].

For the EKF-based direct reconstruction algorithm based on the linear measurement model (32), the number of states $h$ is $n(n+2) N$, where $n$ is the number of compartments, and $N$ is the number of voxels. Typical values of $n, K, N$, and $m$ are tabulated in Table II. Thus, assuming that $2 m^{2} n(n+2) \sim N^{2}$, $2 m n^{2}(n+2)^{2} \sim N^{3 / 2}, m^{3} \sim N^{5 / 2}$, and $n^{3}(n+2)^{3} \sim$ $N$, the computational complexity of direct reconstruction algorithm for one recursion of the EKF algorithm is given by $\mathcal{O}\left(N^{3}\right)+\mathcal{O}\left(N^{3}\right)+\mathcal{O}\left(N^{2}\right)+\mathcal{O}\left(N^{4}\right)$, which is dominated by the $\mathcal{O}\left(N^{4}\right)$ term. For the nonlinear measurement model (31), the Fréchet derivative of $\mathcal{F}$ has to be computed at every recursion, which has a computational complexity of $\mathcal{O}\left(N_{d} N^{2}+N_{s} N^{2}\right)$ [43]. Assuming that $N_{d}$ and $N_{s} \ll N^{2}$, the computational complexity of every recursion of the EKF algorithm using the nonlinear measurement model is $\mathcal{O}\left(N^{4}\right)$. Hence, the EKF-based direct reconstruction algorithms using either the nonlinear or linear measurement model have the computational complexity of $\mathcal{O}\left(N^{4}\right)$.

For the EKF-based voxel-by-voxel construction algorithm that we introduced in [21], the number of states, $h$, is $n(n+2)$ and $m=1$. In this algorithm, the absorption coefficient images are reconstructed prior to pharmacokinetic-rate images. In general, the computational complexity of this step is $\mathcal{O}\left(N^{3} K\right)$ for a linear reconstruction algorithm, 
where $K$ is the number of slow-time steps. Assuming that, $2 n(n+2) \sim N^{1 / 2}, 2 n^{2}(n+2)^{2} \sim N$, and $n^{3}(n+2)^{3} \sim N^{3 / 2}$, the computational complexity of the voxel-by-voxel reconstruction algorithm for one recursion of the EKF is given by $\mathcal{O}\left(N^{3} K\right)+\mathcal{O}\left(N^{3 / 2}\right)+\mathcal{O}\left(N^{2}\right)+\mathcal{O}(N)+\mathcal{O}\left(N^{5 / 2}\right)$, which is dominated by the $\mathcal{O}\left(N^{3} K\right)$ term. For the nonlinear measurement model, the computational complexity of reconstructing the absorption images of fluorophore is $\mathcal{O}\left(N^{3} K p\right)$ where $p$ is the number of iterations performed in Born-type nonlinear iterative reconstruction schemes. Clearly, if the number of slow-time samples multiplied with the iteration number, $K p$, is of higher order than the total number of voxels, $N$, then, the computational complexity of the direct reconstruction algorithm is smaller than that of the voxel-by-voxel algorithm. However, for the current dynamic imaging systems, $K p$, is smaller than $N$. On the other hand, the EKF-based direct reconstruction algorithms offer a number advantages as discussed in Section I, that may justify such an increase in computational requirements.

The algorithm stores three covariance matrices $\mathbf{Q}, \mathbf{R}$, and $\mathbf{Z}$ with size $n N \times n N, m \times m$, and $n(n+1) N \times n(n+1) N$, respectively. For each iteration, the algorithm stores a measurement matrix of size $m \times 1$, stores and updates the error covariance matrix, $\mathbf{P}$, of size $n(n+2) N \times n(n+2) N$, Kalman gain matrix, $\mathbf{H}$, of size $n(n+2) N \times m, \Lambda$ matrix of size $m \times n(n+2) N$, and $\mathbf{J}$ matrix of size $n(n+2) N \times n(n+2) N$. The algorithm also stores all the updates of the concentrations and parameters which are of size $n(n+2) N \times 1$.

\section{Numerical Simulations AND Pharmacokinetic-Rate IMAGE RECONSTRUCTION FROM in vivo BREAST DATA}

We tested the performance of our algorithms using simulated data, and in vivo data acquired from three patients with breast tumors. We first present the numerical simulation results pertaining the convergence and initialization of EKF-based algorithms and performance comparison of the EKF-based direct reconstruction algorithms with that of the voxel-by-voxel reconstruction algorithm presented in [21]. Next, we present the pharmacokinetic-rate images reconstructed from in vivo breast data.

\section{A. Numerical Simulations}

We tested the performance of EKF-based direct reconstruction algorithm based on both the nonlinear (31) and linear (32) measurement models. In the linear model, we computed the matrix $\mathbf{W}$ based on the background $\mu_{a x, m}$, and assumed that it is constant throughout the dynamic update of the fluorophore concentrations. For the nonlinear model, we updated the Fréchet derivative of $\mathcal{F}$, at every iteration of the EKF algorithm based on the $(k \mid k-1)$ th update of the concentration and volume fraction estimates as defined in (35)-(37). In the update of the Fréchet derivatives, we assumed that $\mu_{\text {axe }}$ is constant, and $\mu_{\text {ame }} \gg$ $\mu_{\text {amf }}$. As a result, we computed $G_{m}\left(\boldsymbol{r}_{d}, \boldsymbol{r} ; \omega\right)$ once, and did not update at every recursion, but updated the $\Phi_{x}^{k \mid k-1}\left(\boldsymbol{r}, \boldsymbol{r}_{s} ; \omega\right)$ at every recursion as described in Section V-B.

We used the two-compartment model for the ICG pharmacokinetics described in Section II-B and the light propagation model described in Section III-A.
TABLE III

Physiological VALUES FOR NUMERICAL SimUlations

\begin{tabular}{ll}
\hline Maximum value of $k_{\text {in }}$ & $0.037 \mathrm{sec}^{-1}$ \\
Maximum value of $k_{\text {out }}$ & $0.029 \mathrm{sec}^{-1}$ \\
$k_{\text {elm }}$ & $0.0054 \mathrm{sec}^{-1}$ \\
$v_{e}$ & 0.3 \\
$v_{p}$ & 0.04 \\
$\mu_{\text {axe }}$ & $0.05 \mathrm{~cm}^{-1}$ \\
$\mu_{s x}^{\prime}$ & $8 \mathrm{~cm}^{-1}$ \\
\hline
\end{tabular}

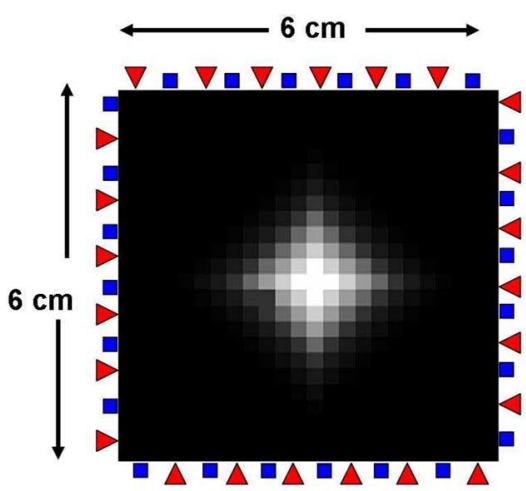

Fig. 4. The source detector configuration used in numerical simulations. Rectangular shapes represent the detectors and the triangular ones represent the sources.

As a prior model, we employed a four-pixel neighborhood model with $\beta=1 / 4$ due to the rectangular nature of the phantom geometry.

1) Data Generation and Phantom Description: Using physiologically relevant values for the pharmacokinetic rates, $k_{\text {in }}$, $k_{\text {out }}, k_{\text {elm }}$, and volume fractions, $v_{e}, v_{p}$, given in Table III, we simulated the boundary measurements, $\Psi(k), k=1, \ldots, K$, for a tissue-like 2-D phantom. The maximum transition rates of $k_{\text {in }}$ and $k_{\text {out }}$ are simulated at the center of the image and smoothly decreased towards the boundaries based on the results given in [18], [21]. Note that since the heterogeneity in the $k_{\text {in }}$ and $k_{\text {out }}$ images is expected to arise from the same physical tumor location and shape, we chose $k_{\text {in }}$ and $k_{\text {out }}$ images to be similar. The fluorescence quantum efficiency and lifetime of ICG are assumed to be constant and set to 0.016 and $0.56 \mathrm{~ns}$, respectively. The modulation frequency was set to $300 \mathrm{MHz}$. The physical dimension of the 2-D phantom was chosen $6 \mathrm{~cm} \times 6$ $\mathrm{cm}$. The image domain was discretized into $24 \times 24$ pixels each of size $0.25 \mathrm{~cm} \times 0.25 \mathrm{~cm} .24$ sources and 24 detectors along the boundary of the phantom were used to generate simulated data, as shown in Fig. 4. The values of $k_{\text {elm }}, v_{e}$, and $v_{p}$ in Table III correspond to the average values of the heterogeneities from the $24 \times 24$ pixel phantom images.

2) Initialization of the EKF-based Algorithms: We studied the effect of the initialization of the covariance matrices $\mathbf{R}$, $\mathbf{Q}$, and $\mathbf{Z}$ in the reconstructed pharmacokinetic-rate images. We chose the initial values of concentrations, pharmacokinetic rates, volume fractions and $\mathbf{R}, \mathbf{Q}$, and $\mathbf{Z}$ matrices within biological limits that lead to an error covariance matrix with minimum norm (trace sum). Let $\mathbf{R}=\alpha_{1} \mathbf{I}, \mathbf{Q}=\alpha_{2} \mathbf{I}$, and $\mathbf{Z}=\alpha_{3} \mathbf{I}$, where $\mathbf{I}$ is an identity matrix, and $\boldsymbol{\alpha}=\left[\begin{array}{lll}\alpha_{1} & \alpha_{2} & \alpha_{3}\end{array}\right]$. Table IV presents 
TABLE IV

Trace Norm of the ERror Covariance MatriX, $\boldsymbol{\alpha}=$ $\left[\begin{array}{lll}0.012 & 0.051 & 0.0025\end{array}\right]$, AND $\boldsymbol{\alpha}=\left[\begin{array}{llll}0.014 & 0.048 & 0.0034\end{array}\right]$ FOR THE LINEAR AND NONLINEAR MEASUREMENT MODELS

\begin{tabular}{lcc}
\hline & LINEAR RECON & NON-LINEAR RECON \\
\hline $\boldsymbol{\alpha} / 10$ & 224.54 & 215.93 \\
$\boldsymbol{\alpha} / 5$ & 152.40 & 143.36 \\
$\boldsymbol{\alpha} / 2$ & 104.34 & 97.34 \\
$\boldsymbol{\alpha}$ & $\mathbf{7 7 . 1 8}$ & $\mathbf{6 3 . 4 5}$ \\
$\mathbf{\alpha} \boldsymbol{\alpha}$ & 107.91 & 95.39 \\
$\mathbf{3} \boldsymbol{\alpha}$ & 148.29 & 140.70 \\
\hline
\end{tabular}

the norm of the error covariance matrix for $k_{\text {in }}$ and $k_{\text {out }}$ for different values of $\boldsymbol{\alpha}$ using both the linear and nonlinear direct reconstruction algorithms for the noise-free measurements. The $\boldsymbol{\alpha}$ value corresponding to the minimum error covariance norm is equal to [0.012 0.0510 .0025$]$, and [0.014 0.0480 .0034 ] for the linear and nonlinear reconstructions, respectively. For the rest of the simulation study, we used the optimal $\boldsymbol{\alpha}$ value to initialize the covariance matrices $\mathbf{Q}$ and $\mathbf{Z}$. The covariance of the measurement noise, i.e., $\mathbf{R}$ was updated based on the additive noise level in the measurements.

3) Performance Comparison: Noise-free Measurements Case-To quantify the difference between the reconstructed and true images, we used the normalized mean square error (NMSE):

$$
\mathrm{NMSE}=20 \log _{10} \frac{\left\|X_{\text {recon }}-X_{\text {true }}\right\|^{2}}{\left\|X_{\text {true }}\right\|^{2}} .
$$

Note that NMSE $=-\mathrm{SNR}$ where SNR stands for signal-tonoise-ratio. For the direct reconstruction algorithm with the nonlinear measurement model, the NMSE for $k_{\text {in }}$ and $k_{\text {out }}$ images are $-19.77 \mathrm{~dB}$ and $-18.49 \mathrm{~dB}$, respectively. For the direct reconstruction algorithm with the linear measurement model, the NMSE for $k_{\text {in }}$ and $k_{\text {out }}$ images are $-18.45 \mathrm{~dB}$ and $-17.65 \mathrm{~dB}$, respectively. Finally, for the voxel-by-voxel construction algorithm, the error for $k_{\mathrm{in}}$ and $k_{\text {out }}$ images are $-16.88 \mathrm{~dB}$ and $-15.90 \mathrm{~dB}$, respectively.

Fig. 5(a) and Fig. 6(a) show the phantom images of the pharmacokinetic-rates $k_{\text {in }}$ and $k_{\text {out }}$. Fig. 5(b) and Fig. 6(b) display the corresponding $k_{\text {in }}$ and $k_{\text {out }}$ images reconstructed by the EKF-based direct reconstruction algorithm using the nonlinear measurement model. Fig. 5(c) and Fig. 6(c) display the corresponding $k_{\text {in }}$ and $k_{\text {out }}$ images obtained by the direct reconstruction algorithm using the linear measurement model. Fig. 5(d) and Fig. 6(d) display the corresponding $k_{\text {in }}$ and $k_{\text {out }}$ images reconstructed by the voxel-by-voxel algorithm that we introduced in [21]. We observe that there is a good agreement between the true and the estimated images in terms of the localization of the heterogeneities. In all three reconstruction algorithms, the center of the heterogeneity (the location corresponding to the maximum value) is consistent with the ones in the original phantom images.

Noisy Measurements Case-Next, we studied the effect of the measurement noise in the performance of the direct reconstruction algorithms and compared it with that of the voxel-byvoxel algorithm. We added zero-mean white Gaussian noise

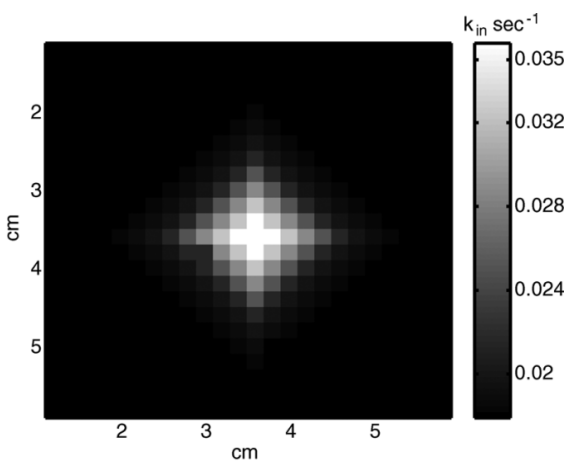

(a)

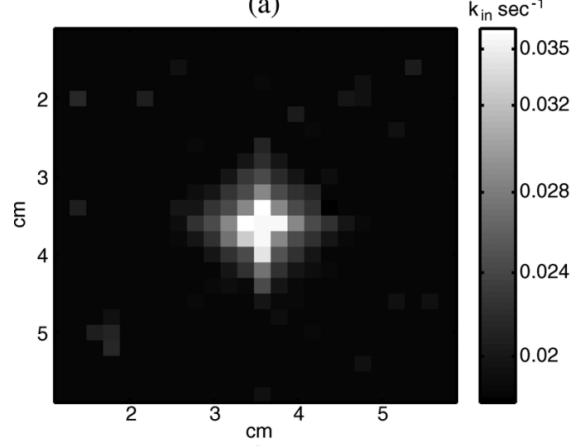

(b)

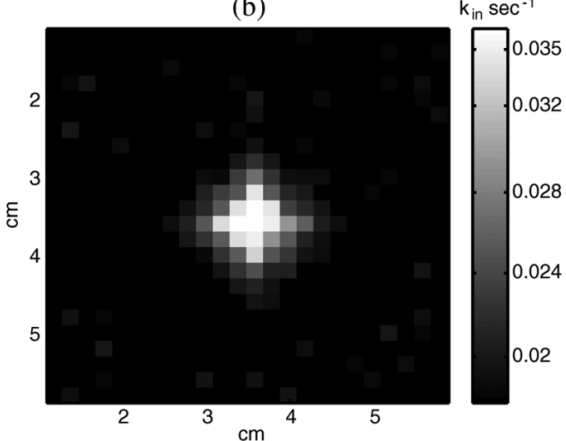

(c)

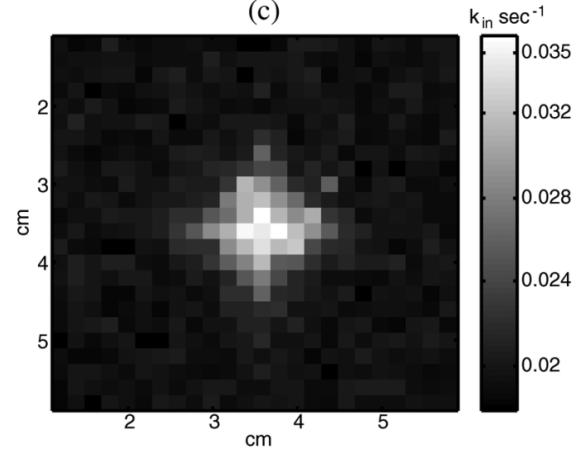

(d)

Fig. 5. Pharmacokinetic-rate images of $k_{\text {in }}$ for three different reconstruction algorithms. (a) Original phantom image. (b) EKF-based direct reconstruction using the nonlinear measurement model. (c) EKF-based direct reconstruction using the linear measurement model. (d) EKF-based voxel-by-voxel reconstruction.

with standard deviation equal to $5 \%-15 \%$ of the average value of the measurements with a step size of $2.5 \%$. We generated 15 realizations of the Gaussian noise at each level and determined the NMSE based on 15 different realizations of noise. Fig. 7(a) and (b) shows the NMSE versus the measurement noise for five different noise levels for $k_{\text {in }}$ and $k_{\text {out }}$ images, respectively. Clearly, the NMSE in the reconstructed $k_{\text {in }}$ and $k_{\text {out }}$ im- 

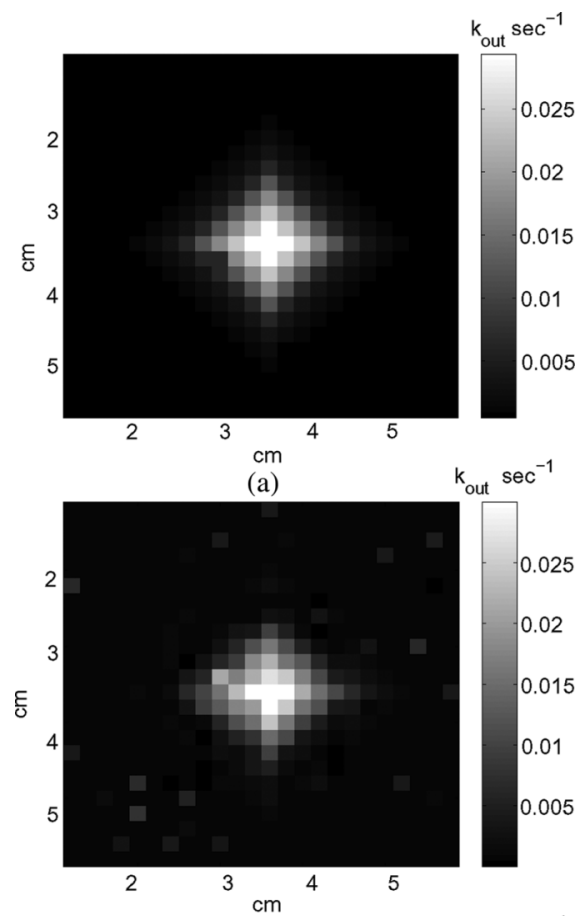

(b)

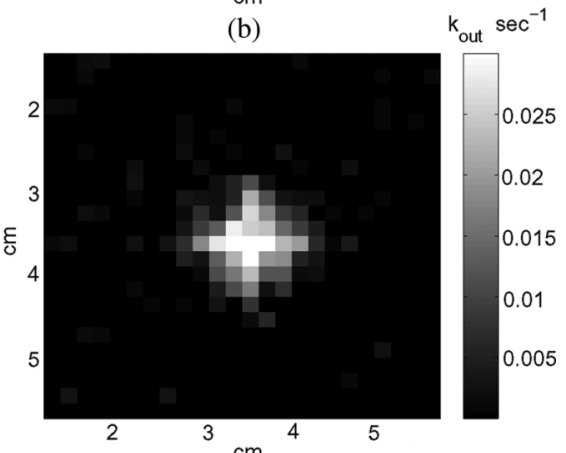

(c)

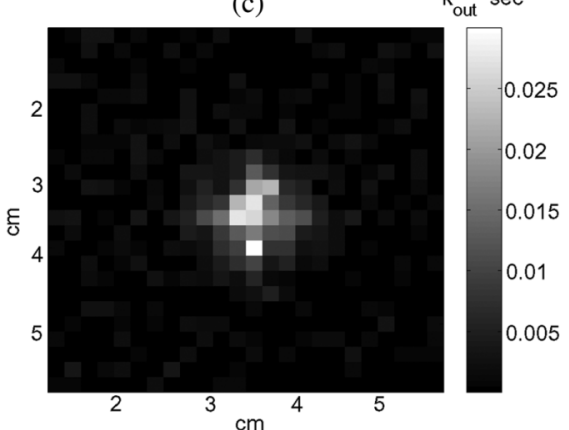

(d)

Fig. 6. Pharmacokinetic-rate images of $k_{\text {out }}$ for three different reconstruction algorithms. (a) Original phantom image. (b) EKF-based direct reconstruction using the nonlinear measurement model. (c) EKF-based direct reconstruction using the linear measurement model. (d) EKF-based voxel-by-voxel reconstruction.

ages increases as the measurement noise increases. The pharmacokinetic-rate images obtained with the direct reconstruction algorithm (both linear and nonlinear cases) together with the $a$ prior information results in smaller error values as compared to the voxel-by-voxel reconstruction algorithm. Additionally, the direct reconstruction algorithm using the nonlinear measurement model results in smaller error values as compared to the

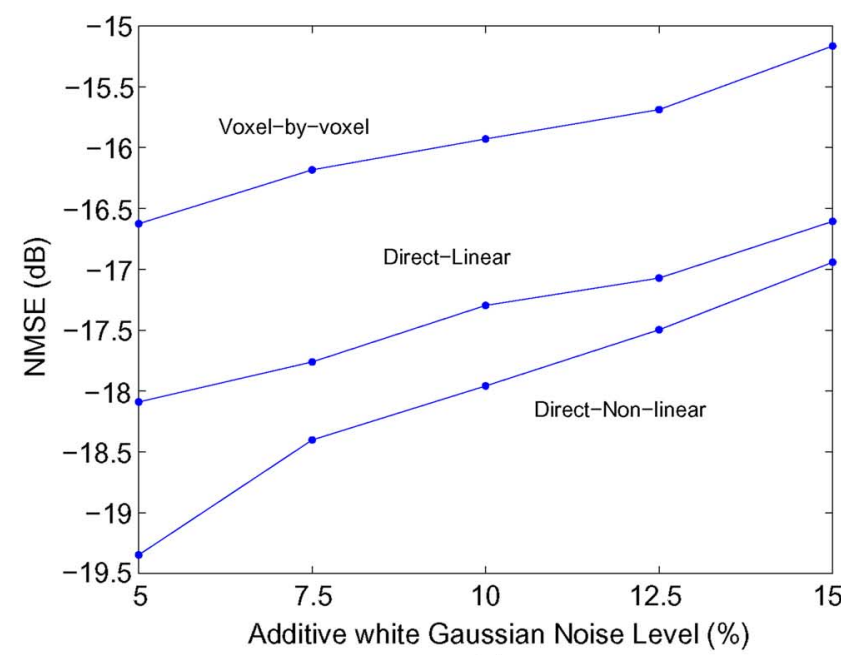

(a)

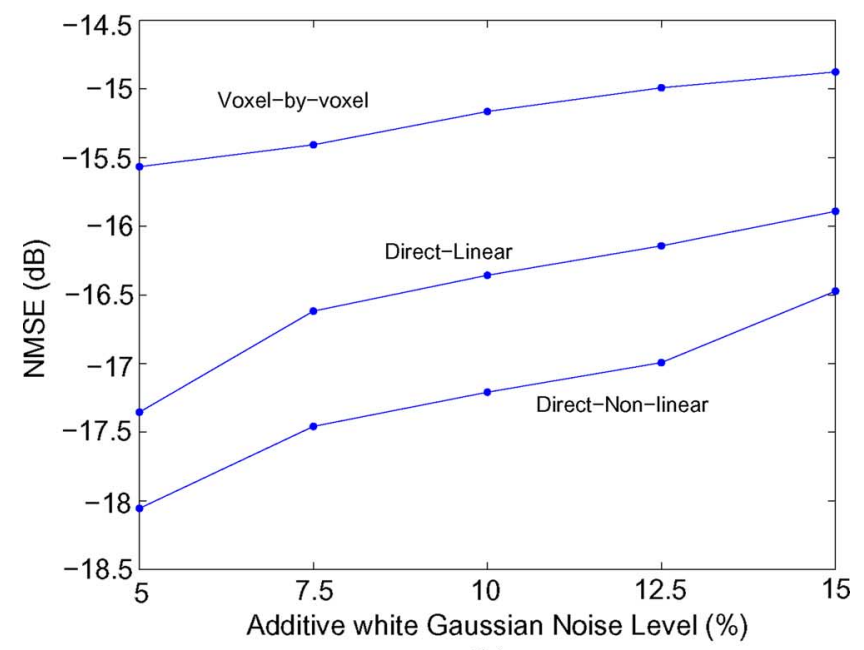

(b)

Fig. 7. NMSE vs measurement noise levels for the direct and voxel-by-voxel reconstruction algorithms (a) $k_{\text {in }}$ images (b) $k_{\text {out }}$ images.

direct reconstruction algorithm based on the linear measurement model.

4) Convergence of the EKF-Based Algorithms: We checked the convergence of the EKF algorithm by studying the $L^{2}$-norm of the residuals of the measurements with respect to the iteration number for the choice of the $\mathbf{R}, \mathbf{Q}$, and $\mathbf{Z}$ matrices described in Section VI-A-2. Note that the norm of the residuals are normalized so that the maximum value is equal to unity for ease of comparison. Fig. 8 shows the normalized $L^{2}$-norm of the residuals versus the iteration number. The decrease in the norm of the residuals confirms the convergence of the EKF algorithm for the choice of $\mathbf{R}, \mathbf{Q}$, and $\mathbf{Z}$ matrices. We see that in the nonlinear measurement model produces smaller residual norms than that of the linear measurement model. We also observe that the norm of the residuals for the nonlinear reconstruction decays at a faster rate than that of the voxel-by-voxel and linear reconstruction. These can be attributed to the more accurate representation of light propagation and the update of the Fréchet derivatives at the prediction step of the EKF-based nonlinear direct reconstruction algorithm. 


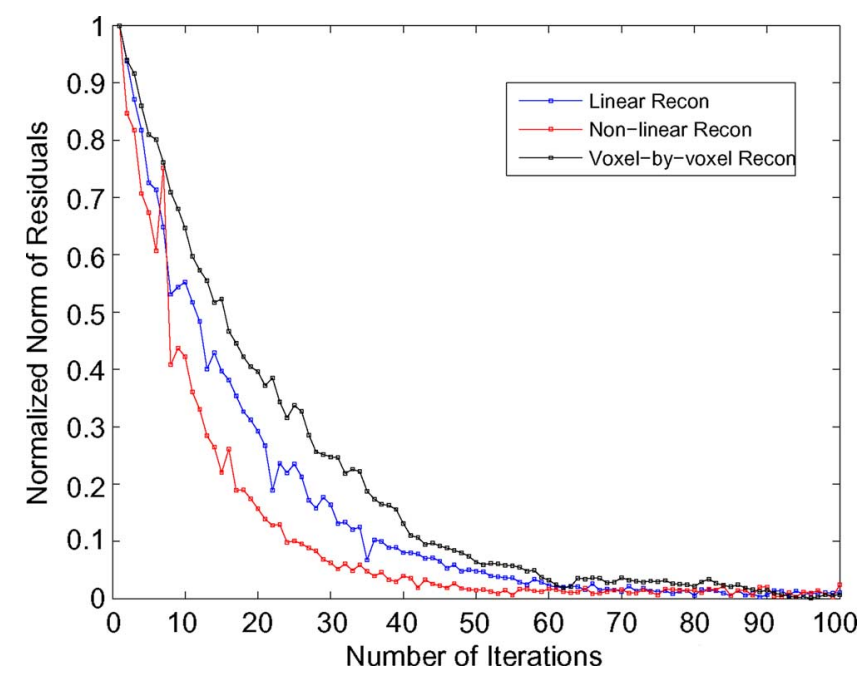

Fig. 8. The normalized $L^{2}$-norm of the residuals versus the number of iterations for the linear and nonlinear direct and voxel-by-voxel reconstruction algorithms in the simulation study.

\section{B. Pharmacokinetic-Rate Images Reconstructed From in vivo Breast Data}

1) Apparatus and Data Collection Protocol: We used in vivo breast data acquired by a continuous wave (CW) NIR imaging apparatus to reconstruct the pharmacokinetic-rate images of ICG. The apparatus has 16 light sources and 16 detectors located on a circular holder at an equal distance from each other with $22.5^{\circ}$ apart. Sources and detectors were collocated and were in the same plane. The breast was arranged in a pendular geometry with the source and detector probes gently touching its surface. A set of measurement for each source was collected at every $500 \mathrm{~ms}$. The total time for the whole scan of the breast including 16 sources and 16 detectors was $8.8 \mathrm{~s}$. The detectors used the same positions as the sources to collect the light originating from one source at a time. We used only the measurements from the farthest 11 detectors with high signal-to-noise ratio in image reconstruction. This resulted in approximately 115 viable measurements out of 256 measurements collected at each time instant. ICG was injected intravenously by bolus with a concentration of $0.25 \mathrm{mg}$ per kg of body weight. Data acquisition started before the injection of ICG and continued for $10 \mathrm{~min}$.

2) Patient Information: Three patients with different tumor types were included in the study. First case, Case 1, is a fibroadenoma, which corresponds to a mass estimated to be $1-2 \mathrm{~cm}$ in diameter, and located $1 \mathrm{~cm}$ below the skin at 6-7 o'clock. Second case, Case 2, is an adenocarcinoma corresponding to a tumor estimated to be $2-3 \mathrm{~cm}$ in diameter, and located approximately $2 \mathrm{~cm}$ below the skin at 4-5 o' clock. Third case, Case 3, is an invasive ductal carcinoma, which corresponds to a mass estimated to be $3-4 \mathrm{~cm}$ in diameter, and located $2 \mathrm{~cm}$ below the skin at 6 o'clock. Diagnostic information was obtained by biopsy after data acquisition. A more detailed explanation of the apparatus, the data collection protocol and tumor information can be found in [20].

3) Compartmental Model and EKF-Based Reconstruction: We used a two-compartment model for the ICG pharmacokinetics as described in (3)-(5). We combined the two-compart-

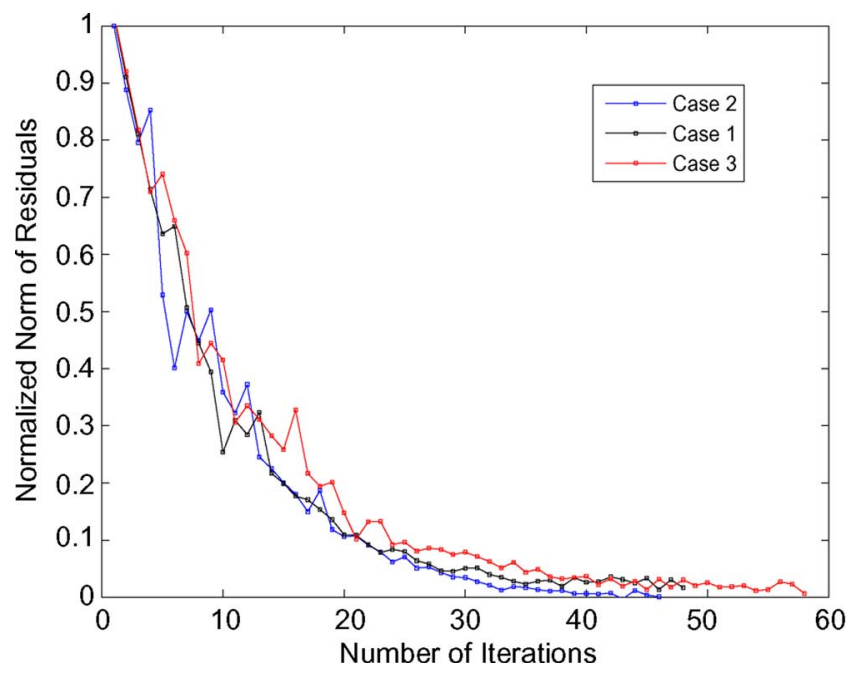

Fig. 9. The $L^{2}$-norm of the residuals versus the number of iterations for the direct linear reconstruction using the in vivo breast data.

ment model with the linear measurement model (16). For computational tractability, we used a 2-D diffusion model for image reconstruction. (See [44]-[47] for a detailed discussion of errors resulting from using a 2-D diffusion model for 3-D light propagation in breast tissue.) We made the following simplifying assumptions: The diffusion coefficient $D_{x}$ is constant and is equal to $0.0416 \mathrm{~cm}$. The endogenous absorption coefficients at the excitation and emission wavelength are approximately the same, $\mu_{\text {ame }} \approx \mu_{\text {axe. }}$. Thus, we determined $G_{m}^{e}$ and $\phi_{x}^{e}$ based on (7) using the excitation measurements prior to ICG injection. We next set the left-hand side of (16) to the excitation measurements after the ICG injection and reconstructed two-dimensional ICG pharmacokinetic-rate and concentration images based on (30) and (32). The resulting measurement model is known as the differential diffuse optical tomography model. A more detailed description of the model can be found in [20], [21], and [48]. As a prior model, we employed a six-pixel neighborhood model with $\beta=1 / 6$ due to the circular nature of the data acquisition geometry. The covariance matrices $\mathbf{R}, \mathbf{Q}$ and $\mathbf{Z}$ were set to the multiples of identity, i.e., $\mathbf{R}=\alpha_{1} \mathbf{I}, \mathbf{Q}=\alpha_{2} \mathbf{I}, \mathbf{Z}=\alpha_{3} \mathbf{I}$ where $\boldsymbol{\alpha}=\left[\begin{array}{lll}0.017 & 0.041 & 0.0013\end{array}\right], \boldsymbol{\alpha}=\left[\begin{array}{lll}0.021 & 0.072 & 0.0029\end{array}\right]$, and $\boldsymbol{\alpha}=\left[\begin{array}{ll}0.030 & 0.0780 .0042\end{array}\right]$ for Case 1, 2, and 3, respectively. We determined these values empirically as described in Section VI-A2.

4) Results: The resulting ICG pharmacokinetic-rate images are shown in Figs. 10-12. The images show that there is a good agreement with the location of the heterogeneity in the images and the physical location of the tumors in axial direction. Additionally, we reconstructed the ICG concentration images for plasma and the EES compartments. Fig. 13(a)-Fig. 18(c) show the ICG concentration in plasma and the EES for three different time instants for Case 1, 2, and 3, respectively. We observed that the pharmacokinetic rates and ICG concentrations in plasma and the EES compartments are higher around the tumors agreeing with the hypothesis that around the tumor region ICG leaks out of compromised capillaries of tumor vessels [49], [50].

Fig. 9 shows the normalized $L^{2}$-norm of the measurements versus the number of iterations for the direct reconstruction al- 

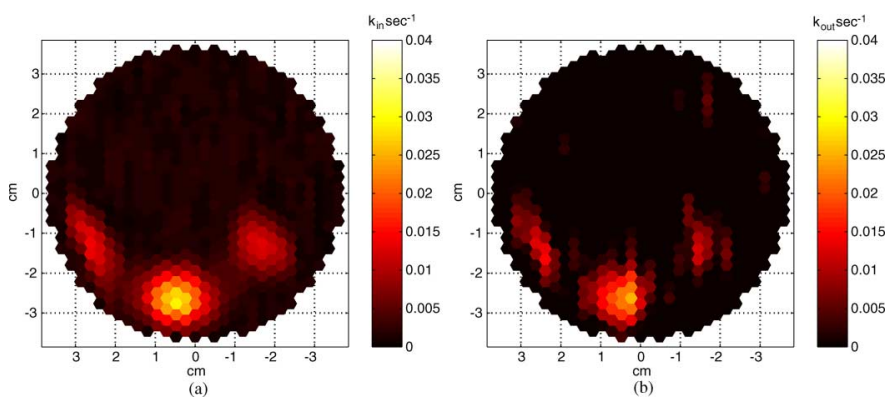

Fig. 10. Direct reconstruction of pharmacokinetic-rate images of (a) $k_{\text {in }}$, (b) $k_{\text {out }}$. Case 1: Fibroadenoma corresponding to a mass of $1-2 \mathrm{~cm}$ in diameter located at 6-7 o'clock.
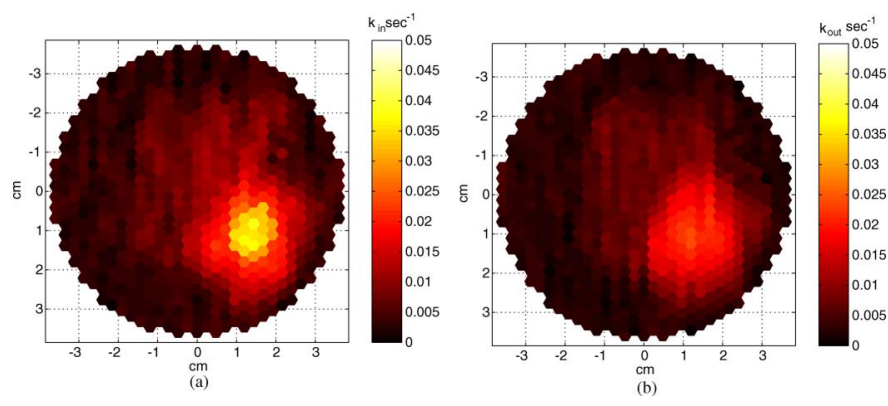

Fig. 11. Direct reconstruction of pharmacokinetic-rate images of (a) $k_{\text {in }}$, (b) $k_{\text {out }}$. Case 2: Adenocarcinoma corresponding to a mass of $2-3 \mathrm{~cm}$ in diameter located at 4-5 o'clock.
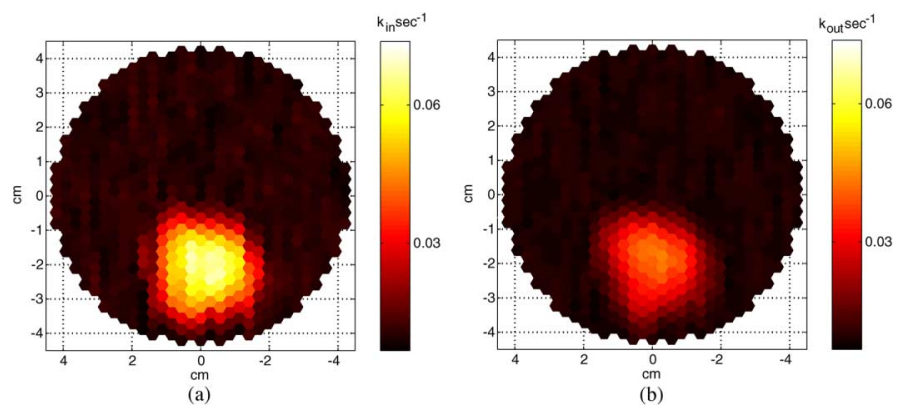

Fig. 12. Direct reconstruction of pharmacokinetic-rate images of (a) $k_{\text {in }}$, (b) $k_{\text {out }}$. Case 3: Ductal carcinoma corresponding to a mass of 3-4 cm in diameter located at 6 o'clock.

gorithm using a linear measurement model. The decrease in the norm of the residuals confirms the convergence of the EKFbased reconstruction algorithm and the goodness of the fit of the two-compartment model for the ICG pharmacokinetics in breast tissue.

Although the number of patient data is limited, our results indicate that the pharmacokinetic-rate imaging may provide new approaches to evaluate and improve breast cancer diagnosis, staging, and treatment monitoring. Such approaches may include extraction of new quantitative features from ICG pharmacokinetic-rate images, and statistical analysis of spatial distribution of pharmacokinetic rates.

\section{CONCLUSION}

In this paper, we presented a new method for the reconstruction of spatially resolved pharmacokinetic rates, volume fractions and concentrations in different compartments for optical
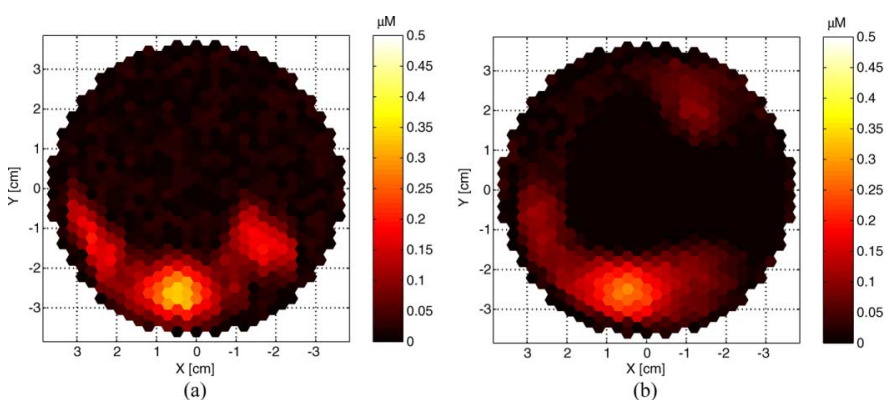

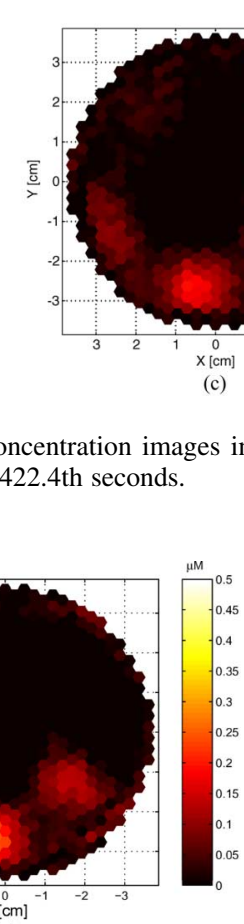

(a)
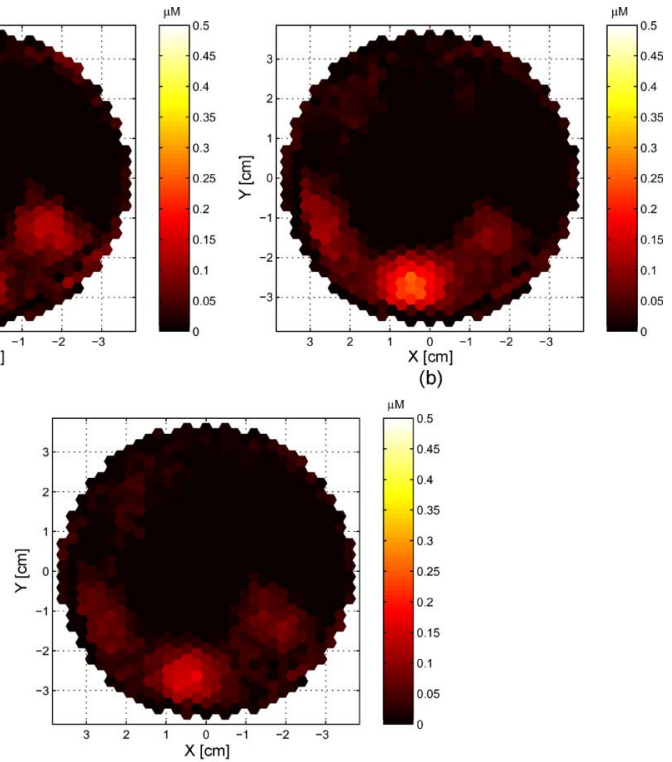

(c)

Fig. 14. ICG concentration images in the EES for Case 1 at (a) 246.4th, (b) 334.4th, and (c) 422.4th s.

fluorophores directly from dynamic NIR boundary measurements. We first derived a mathematical model that maps the boundary measurements to the spatially resolved pharmacokinetic rates and volume fractions. For this, we combined the compartmental modeling with the diffusion approximation to derivative transfer equation to model NIR light propagation in tissue. We next formed a state-space model and introduced a spatio-temporal prior model on the pharmacokinetic-rate and volume fraction images and addressed the resulting image formation problem by the EKF framework. We discussed the initialization of our algorithms and analyzed their computational complexity. 


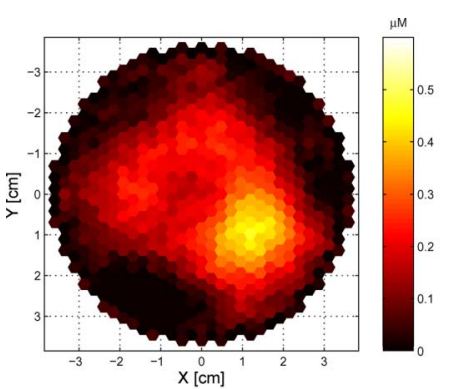

(a)

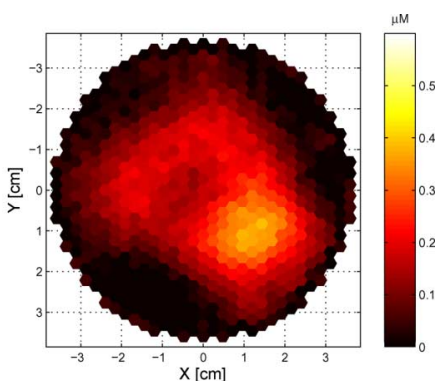

(b)

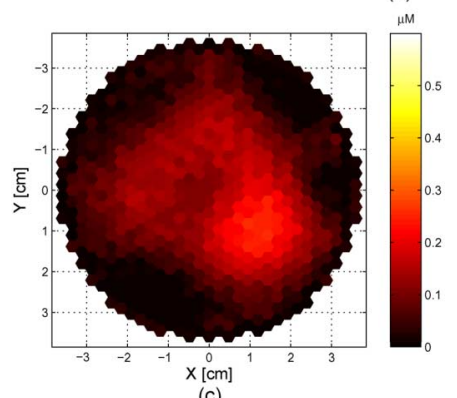

Fig. 15. ICG concentration images in plasma for Case 2 at (a) 228.8th, (b) 316.8th, and (c) 404.8th s.

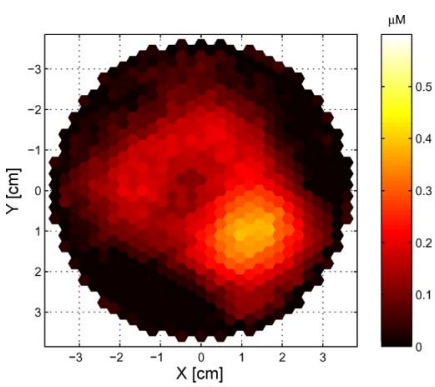

(a)

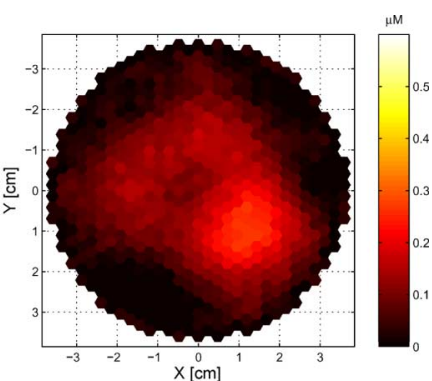

(b)

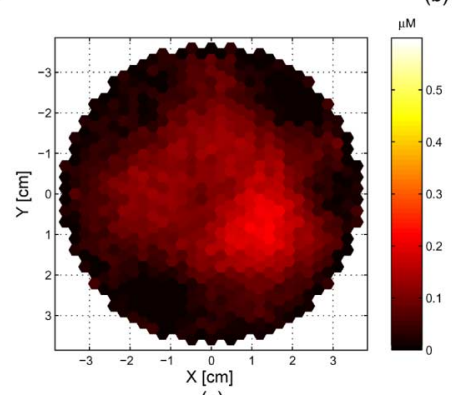

(c)

Fig. 16. ICG concentration images in the EES for Case 2 at (a) 228.8th, (b) 316.8 th, and (c) 404.8th s.

We performed two sets of reconstructions, first set is based on a numerical phantom and the second set is based on in vivo data obtained from patients with breast tumors. The first set demonstrates the performance of the proposed algorithms under different noise levels and compares them with the voxel-by-voxel reconstruction algorithm that we presented in [21]. Our simulation study shows that there is a good agreement between the true and the estimated images in terms of localization of the heterogeneities. Additionally, the EKF-based direct reconstruction algorithms lead to better performance than the voxel-by-voxel reconstruction algorithm with respect to normalized mean-square error criterion.

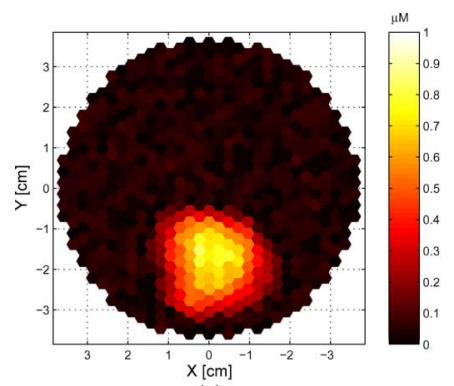

(a)

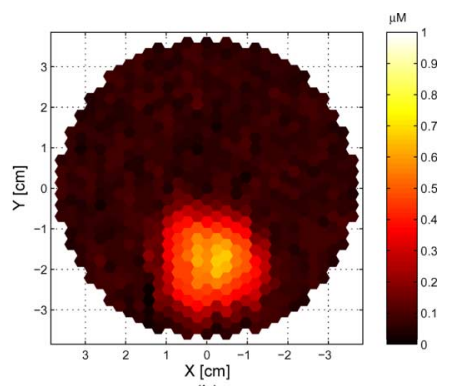

(b)

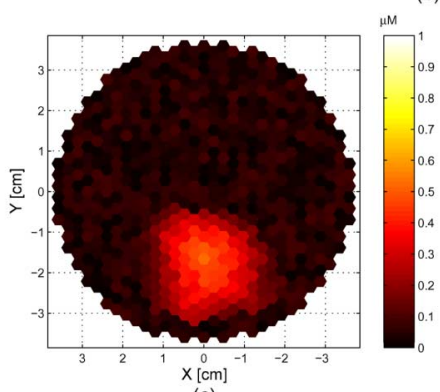

(c)

Fig. 17. ICG concentration images in the plasma for Case 3 at (a) 246.4th, (b) 378.4 th, and (c) 510.4 th s.

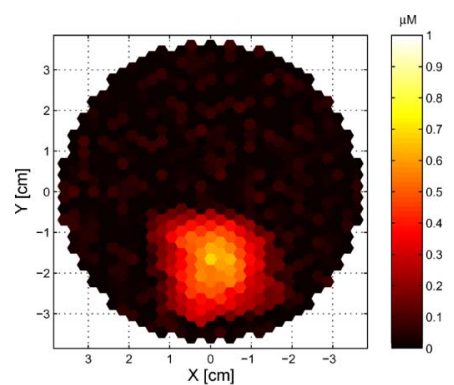

(a)

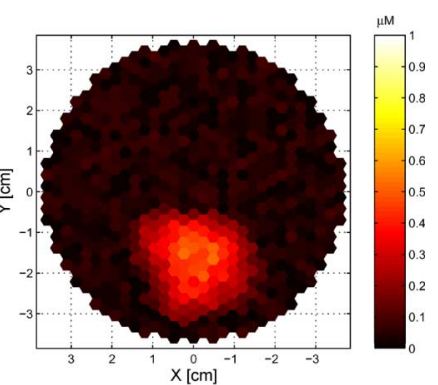

(b)

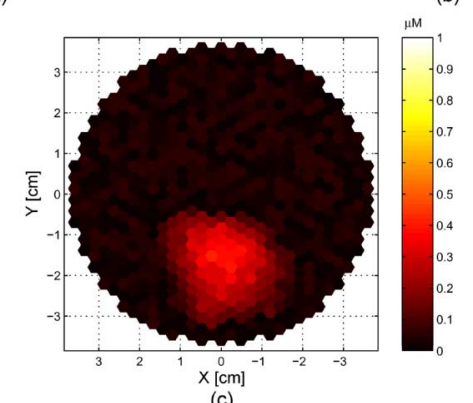

(c)

Fig. 18. ICG concentration images in the EES for Case 3 at (a) 246.4th, (b) 378.4 th, and (c) 510.4 th s.

The improvements in the direct reconstruction technique can be attributed to better use of the temporal correlations between the dynamic NIR measurements by combining spatial and temporal modeling into a single step and the use of prior models on pharmacokinetic-rate images.

Although our simulation study is limited to the time-invariant pharmacokinetic-rate imaging, the compartmental models, and the reconstruction method outlined in our paper is applicable to imaging of time-varying compartmental model parameters.

For the processing of the in vivo breast data, we used a two-compartment model for the ICG pharmacokinetics and combined it with the linear NIR photon propagation model. 
We reconstructed the pharmacokinetic-rate images of ICG using the direct reconstruction method. Our results show that the pharmacokinetic rates are higher around the tumor region agreeing with the fact that permeability increases around the tumor region. Additionally, spatially resolved pharmacokinetic rates coupled with image understanding techniques may lead to new approaches to breast cancer diagnosis, staging and treatment methods.

While the two-compartment model is sufficient to model the ICG pharmacokinetics, higher-order compartmental models may be advantageous for modeling the pharmacokinetics of functionalized optical contrast agents that actively accumulate or activate in diseased tissue [51]-[53]. Additionally, the formalism and the algorithms introduced in this study can be utilized to address the reconstruction of pharmacokinetic-rate images for PET or MR contrast agents by replacing the NIR photon propagation model with the appropriate forward models for PET and MR imaging.

\section{ACKNOWLEDGMENT}

The authors would like to thank their colleagues Dr. X. Intes for discussions on real data analysis, and Dr. M. Guven for fruitful discussions on fluorescence diffuse optical tomography, and Fréchet derivatives.

\section{REFERENCES}

[1] L. A. Bauer, Applied Clinical Pharmacokinetics. New York: McGraw-Hill, 2001.

[2] C. W. Tornoe, "Grey-box PK/PD modeling of insulin," M.S. thesis, DTU, Lyngby, Jun. 28, 2002.

[3] D. H. Anderson, Lecture Notes in Biomathematics. Berlin, Germany: Springer-Verlag, 1983.

[4] J. A. Jacquez, Compartmental Analysis in Biology and Medicine, Kinetics of Distribution of Tracer-Labeled Materials. New York: Elsevier, 1972.

[5] C. C. Martin, R. F. Williams, J. H. Gao, L. D. Nickerson, J. Xiong, and P. T. Fox, "The pharmacokinetics of hyperpolarized xenon: Implications for cerebral MRI,' J. Magn. Reson. Imag., vol. 7, no. 5, pp. 848-854, 1997.

[6] G. R. Moran and F. S. Prato, "Modeling (1H) exchange: An estimate of the error introduced in MRI by assuming the fast exchange limit in bolus tracking," Magn. Reson. Med., vol. 51, no. 4, pp. 816-827, 2004.

[7] R. Srikanchana, D. Thomasson, P. Choyke, and A. Dwyer, "A comparison of pharmacokinetic models of dynamic contrast enhanced MRI," in Proc. 7th IEEE Symp. Computer-Based Med. Syst., 2004, p. 361.

[8] R. E. Carson and K. Lange, "The EM parametric image reconstruction algorithm," J. Am. Statist. Assoc., vol. 80, no. 389, pp. 20-22, 1985.

[9] M. A. Limber, M. N. Limber, A. Cellar, J. S. Barney, and J. M. Borwein, "Direct reconstruction of functional parameters for dynamic SPECT," IEEE Trans. Nucl. Sci., vol. 42, no. 4, pp. 1249-1256, Aug. 1995.

[10] S. R. Meikle, J. C. Matthews, V. J. Cunningham, D. L. Bailey, L. Livieratos, T. Jones, and P. Price, "Parametric image reconstruction using spectral analysis of PET projection data," Phys. Med. Biol., vol. 43, pp. 651-666, 1998.

[11] W. Reutter, G. T. Gullberg, and R. H. Huesman, "Kinetic parameter estimation from attenuated SPECT projection measurements," IEEE Trans. Nucl. Sci., vol. 45, no. 6, pp. 1340-1344, Dec. 1998.

[12] O. Langer, R. Karch, U. Müller, G. Dobrozemsky, A. Abrahim, M. Zeitlinger, E. Lackner, C. Joukhadar, R. Dudczak, K. Kletter, M. Müller, and M. Brunner, "Combined PET and microdialysis for in vivo assessment of intracellular drug pharmacokinetics in humans," $J$. Nucl. Med., vol. 46, no. 11, pp. 1835-1841, 2005.
[13] M. Kamasak, C. A. Bouman, E. D. Morris, and K. Sauer, "Direct reconstruction of kinetic parameter images from dynamic PET data," IEEE Trans. Med. Imag., vol. 24, no. 5, pp. 636-650, May 2005.

[14] D. Hansen, A. Spence, T. Carski, and M. Berger, "Indocyanine green (ICG) staining and demarcation of tumor margins in a rat glioma model," Surg. Neurol., vol. 40, pp. 451-456, 1993.

[15] H. Shinohara, A. Tanaka, T. Kitai, N. Yanabu, T. Inomoto, S. Satoh, Hatano, Y. Yamaoka, and K. Hirao, "Direct measurement of hepatic indocyanine green clearance with near-infrared spectroscopy: Separate evaluation of uptake and removal," Hepatology, vol. 23, pp. 137-144, 1996.

[16] A. ElDeosky, A. Seifalian, M. Cope, D. Delpy, and B. Davidson, "Experimental study of liver dysfunction evaluated by direct indocyanine green clearance using near infrared spectroscopy," Br. J. Surg., vol. 86, pp. 1005-1011, 1999.

[17] B. Alacam, B. Yazici, X. Intes, and B. Chance, "Extended Kalman filtering for the modeling and analysis of ICG pharmacokinetics in cancerous tumors using NIR optical methods," IEEE Trans. IEEE Biomed. Eng., vol. 53, no. 10, pp. 1861-1871, Oct. 2006.

[18] M. Gurfinkel, A. B. Thompson, W. Ralston, T. L. Troy, A. L. Moore, T. A. Moore, J. D. Gust, D. Tatman, J. S. Reynolds, B. Muggenburg, K. Nikula, R. Pandey, R. H. Mayer, D. J. Hawrysz, and E. M. SevickMuraca, "Pharmacokinetics of ICG and HPPH-car for the detection of normal and tumor tissue using fluorescence, near-infrared reflectance imaging: A case study," Photochem. Photobiol., vol. 72, pp. 94-102, 2000.

[19] D. J. Cuccia, F. Bevilacqua, A. J. Durkin, S. Merritt, B. J. Tromberg, G. Gulsen, H. Yu, J. Wang, and O. Nalcioglu, "In vivo quantification of optical contrast agent dynamics in rat tumors by use of diffuse optical spectroscopy with magnetic resonance imaging coregistration," Appl. Opt., vol. 42, no. 1, pp. 2940-2950, Jun. 2003.

[20] X. Intes, J. Ripoll, Y. Chen, S. Nioka, A. G. Yodh, and B. Chance, "In vivo continuous-wave optical breast imaging enhanced with indocyanine green," Med. Phys., vol. 30-6, pp. 1039-1047, 2003.

[21] B. Alacam, B. Yazici, X. Intes, and B. Chance, "Spatially resolved pharmacokinetic-rate images of ICG using near infrared optical methods," J. Phys. Med. Biol., vol. 53-4, pp. 837-859, 2008.

[22] M. J. Eppstein, D. E. Dougherty, T. Troy, and E. M. Sevick-Muraca, "Biomedical optical tomography using dynamic parameterization and Bayesian conditioning on photon migration measurements," Appl. Opt., vol. 38-10, pp. 2138-2150, 2001.

[23] V. Kolehmainen, S. Prince, S. R. Arridge, and J. P. Kaipio, "Stateestimation approach to the nonstationary optical tomography problem," J. Opt. Soc. Am., vol. A 20, pp. 876-889, 2003.

[24] S. Prince, V. Kolehmainen, J. P. Kaipio, M. A. Franceschini, D. Boas, and S. R. Arridge, "Time-series estimation of biological factors in optical diffusion tomography," Phys. Med. Biol., vol. 48, pp. 1491-1504, 2003.

[25] A. B. Milstein, K. J. Webb, and C. A. Bouman, "Estimation of kinetic model parameters in fluorescence optical diffusion tomography," J. Opt. Soc. Am., vol. 22, no. 7, pp. 1357-1368, 2005.

[26] A. B. Milstein, S. Oh, K. J. Webb, C. A. Bouman, Q. Zhang, D. A. Boas, and R. P. Millane, "Fluorescence optical diffusion tomography," Appl. Opt., vol. 42, pp. 3081-3094, 2003.

[27] A. B. Milstein, J. J. Stott, S. Oh, D. A. Boas, R. P. Millane, C. A. Bouman, and K. J. Webb, "Fluorescence optical diffusion tomography using multiple-frequency data," J. Opt. Soc. Am., vol. A 21, pp. 1035-1049, 2004.

[28] C. K. Chui and G. Chen, Kalman Filtering With Real Time Applications. Berlin, Germany: Springer, 1999.

[29] J. Kaipio and E. Somersalo, Statistical and Computational Inverse Problems. New York: Springer, 2004.

[30] E. M. Sevick-Muraca, G. Lopez, T. L. Troy, J. S. Reynolds, and C. L. Hutchinson, "Fluorescence and absorption contrast mechanisms for biomedical optical imaging using frequency-domain techniques," Photochem. Photobiol., vol. 66, no. 55, pp. 55-64, 1997.

[31] S. R. Arridge, "Optical tomography in medical imaging: Topical review," Inverse Problems, vol. 15, pp. R41-R93, 1999.

[32] M. L. J. Landsman, G. Kwant, G. A. Mook, and W. G. Zijlstra, "Lightabsorbing properties, stability, and spectral stabilization of indocyanine green," J. Appl. Physiol., vol. 40, pp. 575-583, 1976.

[33] A. Soubret and V. Ntziachristos, "Fluorescence molecular tomography in the presence of background fluorescence," Phys. Med. Biol., vol. 51, pp. 3983-4001, 2006. 
[34] C. Chen, Linear System Theory and Design. New York: Oxford Univ. Press, 1999.

[35] F. Fedele, J. P. Laible, and M. J. Eppstein, "Coupled complex adjoint sensitivities for frequency-domain fluorescence tomography: Theory and vectorized implementation," J. Computat. Phys., vol. 187-2, pp. 597-619, 2003.

[36] M. J. Eppstein, F. Fedele, J. P. Laible, C. Zhang, A. Godavarty, and E. M. Sevick-Muraca, "A comparison of exact and approximate adjoint sensitivies in fluorescence tomography," IEEE Trans. Med. Imag., vol. 22, no. 10, pp. 1215-1223, Oct. 2003.

[37] M. Boutayeb, H. Rafaralahy, and M. Darouach, "Convergence analysis of the extended Kalman filter used as an observer for nonlinear deterministic discrete-time systems," IEEE Trans. Automatic Control, vol. 42, no. 4, pp. 581-586, 1997.

[38] B. J. Schnekenburger, "An extended Kalman filter as a parameter estimator for linear discrete time systems," M.S. thesis, Univ. NJIT, Newark, NJ, USA, 1988.

[39] L. Ljung, "Asymptotic behavior of the extended Kalman filter as a parameter estimator for linear systems," IEEE Tran. Automa. Control, vol. AC-24, no. 1, pp. 36-50, 1979.

[40] B. F. La Scala and R. R. Bitmead, "Design of an extended Kalman filter frequency tracer," IEEE Trans. Signal Process., vol. 44, no. 3, pp. 739-742, Mar. 1996.

[41] F. C. Trigo, R. Gonzales-Lima, and M. B. P. Amato, "Electrical impedence tomography using the extended kalman filtering," IEEE Trans. Biomed. Eng., vol. 51, no. 1, pp. 72-81, Jan. 2004.

[42] M. J. Gorris, D. A. Gray, and I. M. Y. Mareels, "Reducing the computational load of a Kalman filter," IEEE Electron. Lett., vol. 33-18, pp. 1539-1541, 1997.

[43] M. O'Leary, "Imaging With Diffuse Photon Density Waves," Ph.D. dissertation, Dept. Phys. Astronomy, U. Pennsylvania, Philadelphia, May 1996.
[44] H. Dehghani, B. W. Pogue, J. Shudong, B. Brooksby, and K. D. Paulsen, "Three-dimensional optical tomography: Resolution in small-object imaging," Appl. Opt., vol. 42, no. 16, pp. 3117-3128.

[45] B. W. P. Brian, S. X. Song, T. D. T. Tor, T. O. McBride Troy, J. Shudong, and K. D. P. Keith, "Statistical analysis of nonlinearly reconstructed near-infrared tomographic images: Part I: Theory and simulations," IEEE Trans. Med. Imag., vol. 21, no. 7, pp. 753-764, Jul. 2002.

[46] B. W. P. Brian, S. X. Song, T. D. T. Tor, T. O. McBride Troy, J. Shudong, and K. D. P. Keith, "Statistical analysis of nonlinearly reconstructed near-infrared tomographic images: Part II: Experimental interpretation," IEEE Trans. Med. Imag., vol. 21, no. 7, pp. 764-772, Jul. 2002.

[47] M. E. Eames, Matthew, B. W. Pogue, C. M. Carpenter, and H. Dehghani, "Three dimensional near infrared tomography of the breast," in Proc. SPIE, 2007, vol. 6629, p. 66291K-1.

[48] V. Ntziachristos, B. Chance, and A. Yodh, "Differential diffuse optical tomography," Opt. Express, vol. 5, pp. 230-242, 1999.

[49] J. M. Brown and A. J. Giaccia, "The unique physiology of solid tumors: Opportunities (and problems) for cancer therapy," Cancer Res., vol. 58, no. 7 , pp. 1408-1416, 1998.

[50] H. Hashizume, P. Baluk, S. Morikawa, J. W. McLean, G. Thurston, S. Roberge, R. K. Jain, and D. M. McDonald, "Openings between defective endothelial cells explain tumor vessel leakiness," Am. J. Pathol., vol. 156, no. 4, pp. 1363-1380, 2000.

[51] K. Licha, "Contrast agents for optical imaging," Topics Current Chem., vol. 222, pp. 1-29, 2002.

[52] Y. Chen, G. Zheng, Z. Zhang, D. Blessington, M. Zhang, and H. Li, "Metabolism enhanced tumor localization by fluorescence imaging: In vivo animal studies," Opt. Lett., vol. 28, pp. 2070-2072, 2003.

[53] R. Weissleder, C. H. Tung, U. Mahmood, and A. Bogdanov, "In vivo imaging with protease-activated near-infrared fluorescent probes," Nat. Biotech., vol. 17, pp. 375-378, 1999. 